\title{
Universal activity-based labeling method for ammonia- and alkane-oxidizing bacteria
}

Dimitra Sakoula $\mathbb{D}^{1,3^{凶}}{ }^{\text {}}$, Garrett J. Smith ${ }^{1}$, Jeroen Frank ${ }^{1,2}$, Rob J. Mesman (D) ${ }^{1}$, Linnea F. M. Kop (D) ${ }^{1}$, Pieter Blom ${ }^{1}$, Mike S. M. Jetten ${ }^{1,2}$, Maartje A. H. J. van Kessel ${ }^{1}$ and Sebastian Lücker (iD) ${ }^{1 凶}$

(c) The Author(s) 2021

\begin{abstract}
The advance of metagenomics in combination with intricate cultivation approaches has facilitated the discovery of novel ammonia-, methane-, and other short-chain alkane-oxidizing microorganisms, indicating that our understanding of the microbial biodiversity within the biogeochemical nitrogen and carbon cycles still is incomplete. The in situ detection and phylogenetic identification of novel ammonia- and alkane-oxidizing bacteria remain challenging due to their naturally low abundances and difficulties in obtaining new isolates from complex samples. Here, we describe an activity-based protein profiling protocol allowing cultivation-independent unveiling of ammonia- and alkane-oxidizing bacteria. In this protocol, 1,7-octadiyne is used as a bifunctional enzyme probe that, in combination with a highly specific alkyne-azide cycloaddition reaction, enables the fluorescent or biotin labeling of cells harboring active ammonia and alkane monooxygenases. Biotinylation of these enzymes in combination with immunogold labeling revealed the subcellular localization of the tagged proteins, which corroborated expected enzyme targets in model strains. In addition, fluorescent labeling of cells harboring active ammonia or alkane monooxygenases provided a direct link of these functional lifestyles to phylogenetic identification when combined with fluorescence in situ hybridization. Furthermore, we show that this activity-based labeling protocol can be successfully coupled with fluorescence-activated cell sorting for the enrichment of nitrifiers and alkaneoxidizing bacteria from complex environmental samples, enabling the recovery of high-quality metagenome-assembled genomes. In conclusion, this study demonstrates a novel, functional tagging technique for the reliable detection, identification, and enrichment of ammonia- and alkane-oxidizing bacteria present in complex microbial communities.
\end{abstract}

The ISME Journal (2022) 16:958-971; https://doi.org/10.1038/s41396-021-01144-0

\section{INTRODUCTION}

Autotrophic ammonia- and methane-oxidizing bacteria (AOB and MOB, respectively) [1] are ubiquitous in the environment [2] and are of high biotechnological interest [3-9]. Besides their similar environmental distributions, they share many biochemical, morphological, and physiological characteristics [10]. More specifically, both microbial guilds can perform aerobic oxidation of ammonia and methane due to the substrate promiscuity of their key enzymes, but neither group exhibits growth on the alternative substrate [10-12]. Over the last two decades, metagenomic approaches resulted in the identification of novel groups of ammonia- [13-15] and methaneoxidizing microorganisms [2, 16-18], highlighting that our understanding of the microbial biodiversity within the nitrogen and carbon biogeochemical cycles can still be expanded [19]. However, even when detected in metagenomic datasets, linking a function to a specific microorganism remains challenging, as it often requires tedious and intricate cultivation techniques to isolate these slowgrowing and fastidious microorganisms. Thus, there is an urgent need for robust cultivation-independent methods that provide reliable information regarding the identity and activity of the microorganisms present in complex microbial communities. To achieve this, various in vivo and in vitro activity-based protein profiling (ABPP) protocols have been developed to link detectable marker molecules to catalytically active enzymes with specific functions [20].

ABPP techniques employ bifunctional enzyme probes that feature (i) a reactive group, which covalently binds to the active site and thereby inhibits the enzyme, and (ii) an ethynyl or azide group that allows the attachment of a reporter group (e.g., fluorophores or biotin) to the enzyme via a $\mathrm{Cu}(\mathrm{l})$-catalyzed alkyneazide cycloaddition (CUAAC) reaction [21]. These protocols exhibit high specificity for the targeted enzymes as sufficient accessibility of the bifunctional enzyme probe to the active site and structural and/or chemical similarity with the substrate are necessary [20]. The subsequent use of rapid CuAAC reactions performed under mild, aqueous conditions guarantees minimal unspecific reactivity of the reporters and enables the use of low molecular weight bifunctional probes that can penetrate biological membranes [22-24]. Subsequently, depending on the reporter type, the reporter-conjugated enzyme can be subjected to numerous downstream applications such as fluorescent imaging [25-27], mass spectrometry-based proteomics $[28,29]$, and affinity purification of the labeled proteins [30]. Several microbial proteins have been studied using ABPP protocols so far. These studies provided significant insights into

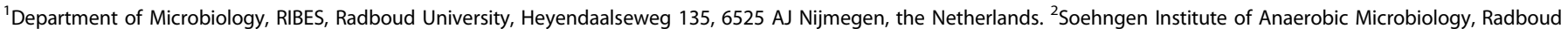

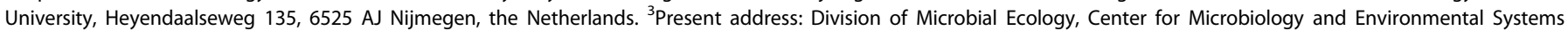
Science, University of Vienna, Althanstraße 14,1090 Vienna, Austria. ${ }^{\circledR}$ email: dimitra.sakoula@univie.ac.at; s.luecker@science.ru.nl 
antibiotic resistance, enzymatic functions in pathogenic bacteria (e.g., serine proteases, kinases, ATPases, fatty acid synthases, glycoside hydrolases) and protein redox dynamics [20]. However, only a limited number of ABPP protocols targeting microbial proteins catalyzing key processes of biogeochemical cycles have been developed [14, 31-33].

Enzymes in the copper-containing membrane monooxygenase (CUMMO) family catalyze diverse reactions, including the oxidation of ammonia, methane, and simple short-chain hydrocarbons [34]. These CuMMO enzymes generally exhibit high genetic, structural, and catalytic similarities [35-37]. AOB and ammonia-oxidizing archaea (AOA) use ammonia monooxygenase (AMO) for the first step of nitrification, the oxidation of ammonia to hydroxylamine [38]. Similarly, the particulate methane monooxygenase (pMMO) present in most $\mathrm{MOB}$ oxidizes methane to methanol [39, 40]. Other hydrocarbon-oxidizing CuMMO (pHMO) enzymes have been reported to catalyze $\mathrm{C}_{2}-\mathrm{C}_{4}$ alkane oxidation in some, taxonomically diverse bacteria [34, 41, 42]. Moreover, some MOB also contain a cytoplasmic diiron-containing soluble methane monooxygenase (sMMO), which is expressed mainly under copper-limited conditions $[43,44]$ and can even constitute the sole methane monooxygenase in some MOB [45]. Although the substrate for both methane monooxygenases is identical, the sMMO shares little structural homology with pMMO or other CuMMO enzymes but rather exhibits a high degree of similarity to known soluble diiron alkane and other hydrocarbon monooxygenases (sHMO) $[37,46]$.

The recent discovery of the novel comammox (complete ammonia oxidation) Nitrospira, featuring a distinct AMO enzyme previously misclassified as an "unusual" pMMO [13, 14], exemplified our still incomplete understanding of the microbial diversity catalyzing ammonia, methane, and simple alkane oxidation. To date, many insights into the distribution, abundance, and activity of ammonia-oxidizing prokaryotes and $\mathrm{MOB}$ in natural environments have been obtained by employing reversible [47-50] and irreversible inhibitors [31, 51-53]. Many terminal $n$-alkynes can be catalytically activated by CuMMO enzymes producing reactive intermediates that bind covalently to the enzyme and act as mechanism-based irreversible inactivators [46, 52, 54-56]. Similarly, alkynes have been established as mechanism-based inactivators of sMMO and other sHMO $[46,56]$. The alkyne 1,7octadiyne $(1,7 \mathrm{OD})$ was recently characterized as a mechanismbased inactivator of the AMO enzyme in Nitrosomonas europaea and was successfully used in in vitro ABPP protocols [31].

In this study, we describe the use of 1,70D in an activity-based labeling protocol for the in situ biotin or fluorescent labeling of bacterial ammonia, methane, and alkane monooxygenases. In combination with transmission electron microscopy, the biotin tagging of $\mathrm{AMO}$ and $\mathrm{PMMO}$ permitted the study of their intracellular distribution. Fluorescent labeling of diverse CuMMO and sHMO enzymes allowed the function-based detection of catalytically active ammonia-, methane-, and alkane-oxidizing microorganisms by fluorescence microscopy, which can be combined with fluorescence in situ hybridization [57] for phylogenetic identification. Moreover, this method can efficiently be used for fluorescence-activated cell sorting (FACS), allowing the enrichment of ammonia-, methane-, and alkane-oxidizing bacteria from complex environmental samples. Together with downstream metagenomics, this facilitates the targeted retrieval of high-quality metagenome-assembled genomes (MAGs) of functionally constrained subpopulations.

\section{MATERIALS AND METHODS \\ Cultivation}

A pure culture of the canonical AOB N. europaea (DSM 28437) was obtained from the Leibniz Institute DSMZ-German Collection of Microorganisms and Cell Cultures (Braunschweig, Germany). N. europaea was grown in batch in mineral salts (MS) medium amended with $10 \mathrm{mM} \mathrm{NH}_{4} \mathrm{Cl}$ [58]. A culture of the AOB Nitrosospira multiformis was maintained in MS medium supplemented with $1 \mathrm{mM}$ ammonium as described by Norton et al. [59]. The comammox strain Nitrospira inopinata was grown as described elsewhere with $1 \mathrm{mM} \mathrm{NH}_{4} \mathrm{Cl}$ [60], and the canonical nitriteoxidizing bacterium Nitrospira moscoviensis $\mathrm{M}-1$ in $\mathrm{MS}$ medium according to Mundiger et al. [61]. Cultures of the MOB Methylotetracoccus oryzae (nitrogen-fixing, Type Ib methanotroph), Methylosinus sporium M29 (Type II methanotroph), Methylocella tundrae (Type II methanotroph containing sMMO only), and Methylacidiphilum fumariolicum (Type III methanotroph) were maintained in batch in nitrate mineral salts (NMS) medium as previously described [17, 62-64]. The propane-oxidizing pHMO-containing Rhodococcus sp. strain ZPP was grown in NMS medium [41] and the butane-degrading sHMO-containing Thauera butanivorans strain Bu-B1211 (DSM 2080) [65] in Brunner mineral medium (DSMZ medium 457). All alkane-oxidizing cultures were inoculated into $20 \mathrm{~mL}$ medium in $120 \mathrm{~mL}$ serum bottles and the headspace was amended with $1 \%(\mathrm{v} / \mathrm{v})$ of methane, propane, or butane, respectively, which were replenished when consumed. A pure culture of the diazotrophic Kyrpidia spormannii FAVT5 was cultivated according to Hogendoorn et al. [66]. Escherichia coli was grown in standard Luria-Bertani broth. An enrichment culture containing the comammox species $\mathrm{Ca}$. Nitrospira nitrosa and $\mathrm{Ca}$. Nitrospira nitrificans was maintained in a sequencing batch bioreactor as reported previously [14]. An enrichment culture of the novel comammox species $\mathrm{Ca}$. Nitrospira kreftii was maintained in a membrane chemostat as described elsewhere [67]. A nitrifying enrichment culture containing canonical $A O B$, canonical and comammox Nitrospira was maintained in a continuous membrane bioreactor fed with MS medium $\left(200 \mathrm{mg} / \mathrm{L} \quad \mathrm{MgSO}_{4} \cdot 7 \mathrm{H}_{2} \mathrm{O}, 30 \mathrm{mg} / \mathrm{L}\right.$ $\mathrm{CaCl}_{2} \cdot 2 \mathrm{H}_{2} \mathrm{O}, 58 \mathrm{mg} / \mathrm{L} \mathrm{NaCl}$ ) supplemented with $0.5 \mathrm{~mL} / \mathrm{L}$ of a trace element stock solution composed of NTA $(15 \mathrm{~g} / \mathrm{L}), \mathrm{ZnSO}_{4} \cdot 7 \mathrm{H}_{2} \mathrm{O}(0.43 \mathrm{~g} / \mathrm{L})$, $\mathrm{CoCl}_{2} \cdot 6 \mathrm{H}_{2} \mathrm{O} \quad(0.24 \mathrm{~g} / \mathrm{L}), \quad \mathrm{MnCl}_{2} \cdot 4 \mathrm{H}_{2} \mathrm{O} \quad(0.99 \mathrm{~g} / \mathrm{L}), \quad \mathrm{CuSO}_{4} \cdot 5 \mathrm{H}_{2} \mathrm{O} \quad(0.25 \mathrm{~g} / \mathrm{L})$, $\mathrm{Na}_{2} \mathrm{MoO}_{4} \cdot 2 \mathrm{H}_{2} \mathrm{O}(0.22 \mathrm{~g} / \mathrm{L}), \mathrm{NiCl}_{2} \cdot 6 \mathrm{H}_{2} \mathrm{O}(0.19 \mathrm{~g} / \mathrm{L}), \mathrm{NaSeO}_{4} \cdot 10 \mathrm{H}_{2} \mathrm{O}(0.021 \mathrm{~g} / \mathrm{L})$, $\mathrm{H}_{3} \mathrm{BO}_{4}(0.014 \mathrm{~g} / \mathrm{L}), \mathrm{CeCl} \cdot 6 \mathrm{H}_{2} \mathrm{O}(0.24 \mathrm{~g} / \mathrm{L})$, and $0.5 \mathrm{~mL} / \mathrm{L}$ of an iron stock solution composed of NTA $(10 \mathrm{~g} / \mathrm{L})$ and $\mathrm{FeSO}_{4}(5 \mathrm{~g} / \mathrm{L})$. The $\mathrm{pH}$ was automatically maintained at 8.0 by dosing $1 \mathrm{M} \mathrm{KHCO}_{3}$. The culture was grown under substrate-limited conditions with $<100 \mu \mathrm{M}$ ammonium, $<20$ $\mu \mathrm{M}$ nitrite, and $250-500 \mu \mathrm{M}$ nitrate supplied to the system. Activated sludge samples were obtained from the aeration tank of a municipal wastewater treatment plant (WWTP) in Groesbeek, The Netherlands $\left(51^{\circ} 45^{\prime} 34.3^{\prime \prime} \mathrm{N} 5^{\circ}\right.$ $57^{\prime} 15.9^{\prime \prime} \mathrm{E}$ )

Unless stated otherwise, biomass from the different pure cultures, bioreactors, and the WWTP sample was harvested by gentle centrifugation $(600 \times g$ for $10 \mathrm{~min})$, washed twice, and subsequently resuspended in MS medium to a final density corresponding to approximately $100 \mu \mathrm{g}$ protein $/ \mathrm{mL}$.

\section{Inactivation of comammox Nitrospira by 1,70D}

To test for the irreversible inactivation of comammox Nitrospira by 1,70D, biomass from the $\mathrm{Ca}$. N. kreftii enrichment culture [67] was harvested and resuspended in $50 \mathrm{~mL}$ MS medium without nitrogen containing $100 \mu \mathrm{M}$ $1,70 D$ ( $99 \%$ purity, Merck KGaA, Darmstadt, Germany) from a stock solution $(75.4 \mathrm{mM})$ in dimethyl sulfoxide (DMSO). Suspensions were incubated for $10 \mathrm{~min}$ in the dark in a shaking incubator $\left(150 \mathrm{rpm}, 24^{\circ} \mathrm{C}\right)$. Subsequently, incubations were supplemented with $\mathrm{NH}_{4} \mathrm{Cl}$ to a final concentration of $350 \mu \mathrm{M}$ and were incubated for $72 \mathrm{~h}$. Control incubations receiving only DMSO were performed in parallel as a negative control. As a positive control for the inhibition of the ammonia oxidation activity, cells were incubated in the presence of $100 \mu \mathrm{M}$ allylthiourea (ATU; $\geq 98 \%$ purity, Merck KGaA, Darmstadt, Germany), which has been shown to inhibit comammox Nitrospira previously [14]. Finally, the specificity of 1,70D as inhibitor of the ammonia-oxidizing activity was verified by incubating comammox Nitrospira cells in the presence of $100 \mu \mathrm{M} 1,70 \mathrm{D}$ and $20 \mu \mathrm{M}$ $\mathrm{NaNO}_{2}$. All incubations were performed in three biological replicates. During incubation, samples for the determination of ammonium, nitrite, and nitrate concentrations were taken every $2 \mathrm{~h}$ for the first $8 \mathrm{~h}$ of incubation, as well as after 24,48 , and $72 \mathrm{~h}$ of incubation.

\section{Analytical methods}

Ammonium concentrations were measured fluorometrically using a modified orthophatal-dialdehyde assay [68]. Nitrite and nitrate concentrations were determined colorimetrically with the Griess reaction [69] as described elsewhere [70]. For the determination of total protein content, cells were lysed using the Bacterial Protein Extraction Reagent (Thermo Fisher Scientific, Waltham, MA, USA) according to the manufacturer's instructions in combination with mild sonication ( $1 \mathrm{~min}, 20 \mathrm{~Hz}$ ). Post lysis, protein concentrations were 
measured using the Pierce bicinchoninic acid protein assay kit (Thermo Fisher Scientific, Waltham, MA, USA) according to the "Enhanced Test-tube" protocol. All fluoro- and colorimetric measurements were performed using a Tecan Spark M10 plate reader (Tecan Trading AG, Männedorf, Switzerland). Methane, propane, and butane concentrations were measured using gas chromatography (HP 5890a, equipped with a flame ionization detector and a Porapak Q column at $80^{\circ} \mathrm{C}$ ).

\section{In situ activity-based fluorescent labeling of ammonia and alkane monooxygenase-containing cells}

Active biomass of the different pure and enrichment cultures, as well as the WWTP sample (see above) was harvested as described above, resuspended in $50 \mathrm{~mL}$ MS medium and incubated with $100 \mu \mathrm{M} 1,7 \mathrm{OD}$ for $30 \mathrm{~m}(150 \mathrm{rpm})$ in the dark. Subsequently, cells were pelleted by gentle centrifugation $(600 \times g$ for $10 \mathrm{~min})$, washed twice in sterile PBS, pH 7.5 and fixed using a $50 \%(\mathrm{v} / \mathrm{v})$ ethanol/PBS solution for $10 \mathrm{~min}$ at room temperature (RT). Fixed biomass was washed once with PBS and subjected to the CuAAC reaction, which was performed in plastic microcentrifuge tubes $(1.5 \mathrm{~mL})$ in a final volume of $250 \mu \mathrm{L}$. Biomass was resuspended in $221 \mu \mathrm{L}$ sterile PBS, mixed with $12.5 \mu \mathrm{L}$ of a $100 \mathrm{mM}$ freshly prepared sodium ascorbate solution ( $\geq 99 \%$ purity, Merck KGaA, Darmstadt, Germany) and $12.5 \mu \mathrm{L}$ of a $100 \mathrm{mM}$ freshly prepared aminoguanidine hydrochloride solution ( $\geq 98 \%$ purity, Merck $\mathrm{KGaA}$ ). A dye mixture containing $1.25 \mu \mathrm{L}$ of a $20 \mathrm{mM} \mathrm{CuSO}_{4}$ solution $(99.99 \%$ purity, Merck $\mathrm{KGaA}$ ), $1.25 \mu \mathrm{L}$ of a $100 \mathrm{mM}$ Tris (3-hydroxypropyltriazolylmethyl)amine solution ( $95 \%$ purity, Merck KGaA), and $0.3 \mu \mathrm{L}$ of $5 \mathrm{mM}$ Azide-Fluor 488 in DMSO ( $290 \%$ purity, Merck KGaA) was incubated in the dark for 3 min. Subsequently, the dye mixture $(2.8 \mu \mathrm{L})$ was added to the CuAAC reaction tubes. The tubes were gently mixed and incubated for $60 \mathrm{~min}$ (RT, in the dark). CuAAC reactions were terminated by harvesting the cells by gentle centrifugation $(600 \times g$ for $10 \mathrm{~min})$. Cell pellets were washed three times with sterile PBS to remove unbound fluorophores and either used directly for downstream analyses, or resuspended in a $50 \%(\mathrm{v} / \mathrm{v})$ ethanol/PBS solution and stored at $-20^{\circ} \mathrm{C}$.

\section{Fluorescence in situ hybridization and microscopy}

Activity-based labeled biomass was hybridized with fluorescently tagged oligonucleotides as described by Daims et al. [71]. Probes used in this study (Supplementary Table S1) were $5^{\prime}$ or $5^{\prime}$ and $3^{\prime}$-labeled with the dyes Cy3 or Cy5 [72]. After hybridization and washing, slides were dried and embedded in DAPI-containing Vectashield antifading mounting medium (\#H-1200, Vector Laboratories Inc., Burlingame, CA). Probe and activityconferred fluorescence was recorded using a Leica TCS Sp8x confocal laser microscope (CLSM; Leica Microsystems B.V., Amsterdam, the Netherlands) equipped with a $405 \mathrm{~nm}$ UV diode and a pulsed white light laser. Images were recorded using $63 \times$ or $100 \times$ oil immersion objectives at a resolution of $1024 \times 1024$ pixels and 8-bit depth.

The spatial distribution of the activity-based fluorescent signal within bacterial cells was investigated using the HyVolution deconvolution module of the Huygens Essential Suite (Scientific Volume Imaging B.V, Hilversum, The Netherlands). N. europaea, $N$. inopinata, $M$. oryzae, and $M$. tundarae were used as representatives of canonical $A O B$, comammox bacteria, and $\mathrm{pMMO}$ and $\mathrm{SMMO}$-only containing MOB, respectively. Images were acquired using a Leica Sp8x CLSM with a $100 \times$ oil immersion objective, a $0.5 \mathrm{AU}$ pinhole size at a resolution of $1024 \times 1024$ pixels, and were deconvolved using the resolution-optimized algorithm of the HyVolution module.

\section{Immunogold localization of the AMO and PMMO enzymes and quantification}

Active cells of $N$. europaea and $M$. oryzae were inactivated using $1,70 D$ as described above, harvested, fixed for $30 \mathrm{~min}$ at RT (2\% PFA, 0.5\% GA in 0.1 $\mathrm{M}$ phosphate buffer $\mathrm{pH} 7)$ and processed for Tokuyasu sectioning [73, 74]. Cells incubated in the presence of DMSO without 1,70D were used as a negative control. The blocks were trimmed to provide a suitable block face. Subsequently, $65-\mathrm{nm}$ sections were cut on a $35^{\circ}$ diamond knife (Diatome, Biel/Bienne, Switzerland) at $-100^{\circ} \mathrm{C}$ in a cryo-ultramicrotome (UC7/FC7 Leica Microsystems, Vienna, Austria), picked up using a mixture of $1 \%$ methylcellulose in $1.2 \mathrm{M}$ sucrose [75] and transferred to 100\# copper grids (Agar Scientific LTD., Essex, UK).

In order to successfully localize the AMO/pMMO enzymes in the bacterial cells, the thawed cryo-sections were subjected to a CuAAC reaction (as described above) using $5 \mathrm{mM}$ biotin-azide in DMSO (>95\% purity, Jena Bioscience GmbH, Jena, Germany) instead of Azide-Fluor 488. Subsequently, immunogold localization of the biotinylated enzymes was performed according to Slot and Geuze (2007) using a rabbit anti-biotin antibody (ab53494, Abcam plc., Cambridge, UK, diluted 1:600 in 1\% BSA-C) and $10 \mathrm{~nm}$ protein A gold (CMC-UMC Utrecht, the Netherlands). Sections were embedded in methylcellulose containing $0.2 \%$ uranyl-acetate before imaging using a JEOL JEM-1400 flash transmission electron microscope, operating at $120 \mathrm{KV}$.

The localization of the immunogold labels was counted in 9-12 cells for each sample and was classified in one out of four classes: (i) resin, (ii) membrane-associated, (iii) cytoplasmic, and (iv) unclear. To this end, circles with a diameter of $15 \mathrm{~nm}$ were drawn around the center of each gold particle. The gold particles themselves had a given diameter of $10 \mathrm{~nm}$ and the antibody-protein A complex accounted for another $10 \mathrm{~nm}$; thus, the epitopes the antibodies were bound to were expected to lie somewhere within these $15 \mathrm{~nm}$ circles. In case gold particles were located in the cytoplasm but with a membrane present within the circle, the label localization was classified as unclear.

The location of the epitopes that classified as "membrane-associated" was investigated in greater detail. More specifically, labels on the periplasmic side of the intracytoplasmic membrane (ICM) compartments were classified as "periplasmic" while labels present on the cytoplasmic side were classified as "cytoplasmic". Labels localized on transversely sectioned membrane stacks or exactly on top of membranes were classified as unclear.

\section{Effect of growth stage on activity-based fluorescent labeling efficiency}

Cells of $N$. europaea and N. multiformis were grown as described above in buffered medium (4.8 g/L HEPES) at pH 8.0 and 7.8, respectively. Control incubations with $N$. europaea and N. multiformis cells incubated in medium without ammonium as well as abiotic controls were performed in parallel. All incubations were performed in two biological replicates. Samples (10 $\mathrm{mL}$ ) were taken regularly to monitor protein content, ammonium concentration, and $\mathrm{pH}$. Additional samples $(50 \mathrm{~mL})$ were subjected to the activity-based labeling protocol as described above. For the quantification of the relative biovolume fractions that depicted AMO labeling, as well as of signal intensity, the activity-based labeling protocol was combined with FISH using an equimolar mixture of the probes Nso190, Nso1225 and NEU653 (Cy3) and EUB338mix (Cy5) (Supplementary Table S1). Subsequently, 15 image-pairs were recorded per sample at random fields of view using a Leica TCS Sp8x CLSM with the same laser intensity, gain (\%) and pinhole settings. The images were imported into the image analysis software daime [76] and evaluated as described elsewhere [77].

\section{Targeted cell sorting of ammonia and alkane monooxygenase-containing bacterial cells}

Biomass from the nitrifying enrichment culture and activated sludge from a municipal WWTP were subjected to the activity-based fluorescent labeling protocol as described above. For both biomasses, three types of control incubations were performed in parallel. These contained (i) incubation with 1,7OD, but no addition of the fluorescent azide-labeled dye in the CUAAC reaction, (ii) DMSO without 1,70D during the initial incubation, with complete subsequent CUAAC reaction, and (iii) DMSO without $1,7 \mathrm{OD}$ during the initial incubation and no addition of the fluorescent azide-labeled dye in the CuAAC reaction. After labeling, large biomass particles and flocs were partially dispersed by mild sonification ( $30 \mathrm{~s}$ at $20 \mathrm{~Hz}$ ) and either stored at $-80^{\circ} \mathrm{C}$ in glycerol-TE (GlyTE) buffer ( 10 $\mathrm{mM}$ Tris- $\mathrm{HCl}, 1 \mathrm{mM}$ EDTA pH 8.0, 5\% v/v glycerol), or processed directly. If frozen, samples were thawed on ice and subsequently subjected to FACS (BD FACSMelody, BD Biosciences, NJ, USA). Based on the initial cell density determined by FACS, samples were diluted up to $1: 4$ with sterile PBS to ensure a final event rate of $<10,000$ events/s. Control incubations, particularly the treatment with dye but without $1,70 D$, were used to determine a gating strategy for removal of artifacts to exclude populations with off-target labeling or passive dye accumulation. Subsequently, DNA was extracted from the bulk-sorted cell clusters, the unsorted control incubations and the initial biomass, followed by metagenomic sequencing.

In addition, we tested for potential biases on the community composition caused by physical and chemical treatment steps that are part of the activitybased protocol. These included potential effects of washing steps, biomass fixation, sonication, and storing conditions. For this purpose, biomass from the nitrifying enrichment culture was subjected to different combinations of physical and chemical treatments occurring during the activity-based staining 
protocol (Supplementary Table S2), followed by DNA isolation and metagenomic sequencing (see below).

\section{DNA extraction and metagenomic sequencing}

Total DNA from all samples was extracted using the DNeasy Blood \& Tissue Kit (Qiagen Ltd., West Sussex, UK) according to the manufacturer's instructions. Genomic sequencing libraries were prepared using the Nextera XT Kit (Illumina, San Diego, CA, USA) following the manufacturer's recommendations using a total of $1 \mu \mathrm{g}$ of input DNA, normalized to a concentration of $0.2 \mathrm{ng} / \mu \mathrm{L}$. The libraries were sequenced on a MiSeq (Illumina, San Diego, CA, USA) with MiSeq Reagent Kit v.3 ( $2 \times 300$ bp, Illumina) according to the manufacturer's instructions.

\section{Metagenome assembly and binning}

Quality-trimming, adapter removal, and contaminant filtering of pairedend sequencing reads for all datasets were performed using BBDuk (BBTools version 37.76; with settings $\mathrm{ktrim}=\mathrm{r}, \mathrm{k}=23, \mathrm{mink}=11$, hdist $=1$, qtrim $=\mathrm{rl}$, trimq $=17, \mathrm{maq}=20$, maxns $=0$, minlen $=150$ ) [78]. Processed reads from all libraries from the same biological sample were de novo coassembled using metaSPAdes v3.11.1 [79] with default settings, which iteratively assembled the metagenome using $k$-mer sizes of $21,33,55,77$, 99 , and $127 \mathrm{bp}$. After assembly, reads were mapped back to the metagenomic contigs for each sample separately using Burrows-Wheeler Aligner (version 0.7.17) [80], employing the "mem" algorithm. The mapping files were processed and converted using SAMtools version 1.9 [81]. Metagenomic binning was performed including all contigs $\geq 1500 \mathrm{bp}$, using differential coverage, sequence composition, and linkage information. To optimize the binning results, five different binning algorithms were used: BinSanity v0.2.6.1 [82], COCACOLA [83], CONCOCT [84], MaxBin 2.0 v2.2.4 [85], and MetaBAT 2 v2.12.1 [86]. The five bin sets were supplied to DAS Tool v1.0 [87] for consensus binning. Bin quality and completeness were assessed based on single-copy marker gene count by CheckM v1.0.7 [88]. Phylogenetic assignment of the final bins was predicted using the classification workflow of GTDB-Tk v0.2.1 [89].

Besides the assembly and automated binning method described above, the sequencing data retrieved from the FACS-sorted activated sludge sample were assembled separately, with subsequent binning of all contigs $\geq 1500$ bp and manual refinement using the anvi'o metagenomics workflow [90]. Here, contigs not clustering with the rest of the bin, based on sequence composition or coverage, were inspected individually in the assembly graph using Bandage v0.8.1 [91]. In addition, genes present on these contigs were compared against the NCBI non-redundant nucleotide database using BLASTx [92]. Contigs without connections in the assembly graph and containing genes not matching the taxonomic affiliation of the bin were removed from the respective MAG. DRAM was used to annotate these MAGs and assess their quality to conform to standards [93]. MAGs were clustered with the automated bins using dRep (v2.0.0.) with a $99 \%$ identity cutoff [94]

\section{Marker gene recovery and read recruitment}

Genes on assembled contigs $\geq 1500$ bp were identified by Prodigal [95]. The translated amino acid sequences were mined using hidden Markov models (HMM) available from the Pfam database to extract the assembled genes for AMO subunit A (amoA, PF02461) and RNA polymerase beta subunit $(r p o B$, PF04563) $[96,97]$. Genes with a sequence score less than 20 or an $e$ value greater than $1 \mathrm{e}-5$ were inspected manually to remove falsepositives. Amino acid sequences of identified CuMMO genes were aligned with the full-length representatives used to build the HMM using MAFFT (v7.397) [98]. The alignment was manually modified to remove partial sequences, including some references, and gapped regions at both ends were trimmed to the first position at which all references contained a residue, resulting in 113 sequences (98 references) and 227 total alignment positions. Phylogenetic reconstruction of was performed using RAxML (v8.2.10) [99] with rapid bootstrapping (100 bootstraps) and determining the best-scoring maximum-likelihood tree using the Gamma matrix and GTR substitution model. For both marker gene sets, corresponding nucleotide sequences $>50 \%$ of the gene length were used to quantify read recruitment using BBMap (BBTools version 37.76 idfilter $=0.99$, pairedonly $=\mathrm{t}$, ambiguous = random) [78]. Read recruitment per gene and sample was normalized by gene length and sequencing depth as paired reads per kilobase gene length per million reads mapped to the full assembly. $\mathrm{R}$ was used to normalize read abundances, calculate correlations, and for visualization [100, 101].

\section{RESULTS}

Inhibition of ammonia oxidation in comammox by 1,70D

The ability of 1,7OD to act as an irreversible and mechanism-based inactivator of the AMO enzyme in the canonical AOB N. europaea was previously demonstrated [31]. Similarly, diverse CuMMOs and sHMOs including methane monooxygenases were shown to be partially inhibited by alkynes [46, 56, 102]. However, comammox Nitrospira harbor a phylogenetically distinct AMO enzyme [13, 14, 36]. Thus, to test the efficacy of inactivation of the comammox AMO by 1,7OD, inhibition of ammonia oxidation activity was determined in the $\mathrm{Ca}$. $\mathrm{N}$. kreftii enrichment culture, which contained comammox Nitrospira as the sole ammonia-oxidizing microorganism [67].

In the absence of 1,70D, the culture oxidized $350 \mu \mathrm{M}$ ammonium in $48 \mathrm{~h}$ to nitrate without intermediate nitrite accumulation. In contrast, addition of $100 \mu \mathrm{M}$ 1,70D resulted in a rapid and complete inhibition of the ammonia-oxidizing activity, which was comparable to the inhibitory effect of $100 \mu \mathrm{M}$ ATU (Fig. 1a, b). No influence of 1,70D or ATU on the nitrite-oxidizing activity of the culture was detected (Fig. 1c, d).

\section{In situ fluorescent labeling of ammonia and alkane monooxygenases}

We adapted the activity-based AMO labeling protocol developed by Benett et al. [31] to allow the in situ fluorescent labeling of ammonia-, methane-, and other alkane-oxidizing bacteria. Biomass of different CUMMO or sHMO-containing microorganisms was incubated with $100 \mu \mathrm{M} 1,7 \mathrm{OD}$, which leads to the formation of stable 1,7OD-enzyme complexes within the bacterial cells [31, 52]. After $\mathrm{EtOH}$ fixation, the inactivated biomass was subjected to a highly specific CuAAC reaction to enable the covalent coupling of the alkyne-labeled enzymes to an azide-labeled Fluor 488 dye, which resulted in the fluorescent labeling of AMO, pMMO, sMMO, pHMO, and sHMO-containing microorganisms (Fig. 2 and Supplementary Figs. S1 and S2).

The efficiency and specificity of the activity-based labeling was verified using pure cultures of the following nitrifying microorganisms: (i) N. europaea and (ii) N. multiformis, two well-studied canonical AOB; (iii) $N$. inopinata, the only comammox bacterium available as pure culture [60]; (iv) N. moscoviensis, a canonical nitrite-oxidizing bacterium; and (v) Nitrosocosmicus franklandus, an ammoniaoxidizing archaeon. Although in most cultures not all cells appeared to be stained, efficient staining of the N. europaea, N. multiformis, and $N$. inopinata cells was observed when 1,7OD-treated cells were subjected to the CuAAC reaction (Fig. 2a, b and Supplementary Fig. S2c). In contrast, no fluorescent signal was obtained when the cells were not treated with 1,7OD (Supplementary Fig. S1a, b), in canonical nitrite-oxidizing N. moscoviensis (Supplementary Fig. S1j), or in heterotrophic E. coli cells (Supplementary Fig. S1k). However, N. franklandus cells also did not show any fluorescent labeling (Supplementary Fig. S1i), indicating that the activity-based protocol was not able to label the archaeal AMO enzyme.

It has been reported that pMMOs and AMOs exhibit similar inhibition patterns [35] and that both particulate and soluble $(\mathrm{p} / \mathrm{s})$ $\mathrm{MMO}$ and $\mathrm{p} / \mathrm{sHMO}$ enzymes can be inactivated by acetylene as well as other alkyne compounds $[46,52,56,103]$. However, the effect of $1,70 D$ on methane- and other alkane-oxidizing enzyme activities has not been studied yet. To test whether the activitybased labeling protocol with 1,7OD could also label p/sMMOs and $\mathrm{p} / \mathrm{sHMOs}$, the following pure cultures were subjected to our labeling protocol: (i) $M$. oryzae, a type $\mathrm{lb}$ methanotrophic gammaproteobacterium [64] and (ii) $M$. sporium, a type II methanotrophic alphaproteobacterium [62], both encoding pMMO; (iii) M. fumariolicum, an acidophilic methane oxidizer of the phylum Verrucomicrobia with multiple pMMO copies [17]; (iv) M. tundrae, an alphaproteobacterial methanotroph encoding only sMMO [63]; (v) Rhodococcus sp. strain ZPP, an ethane- and propane-oxidizing member of the Actinobacteria containing both 
a
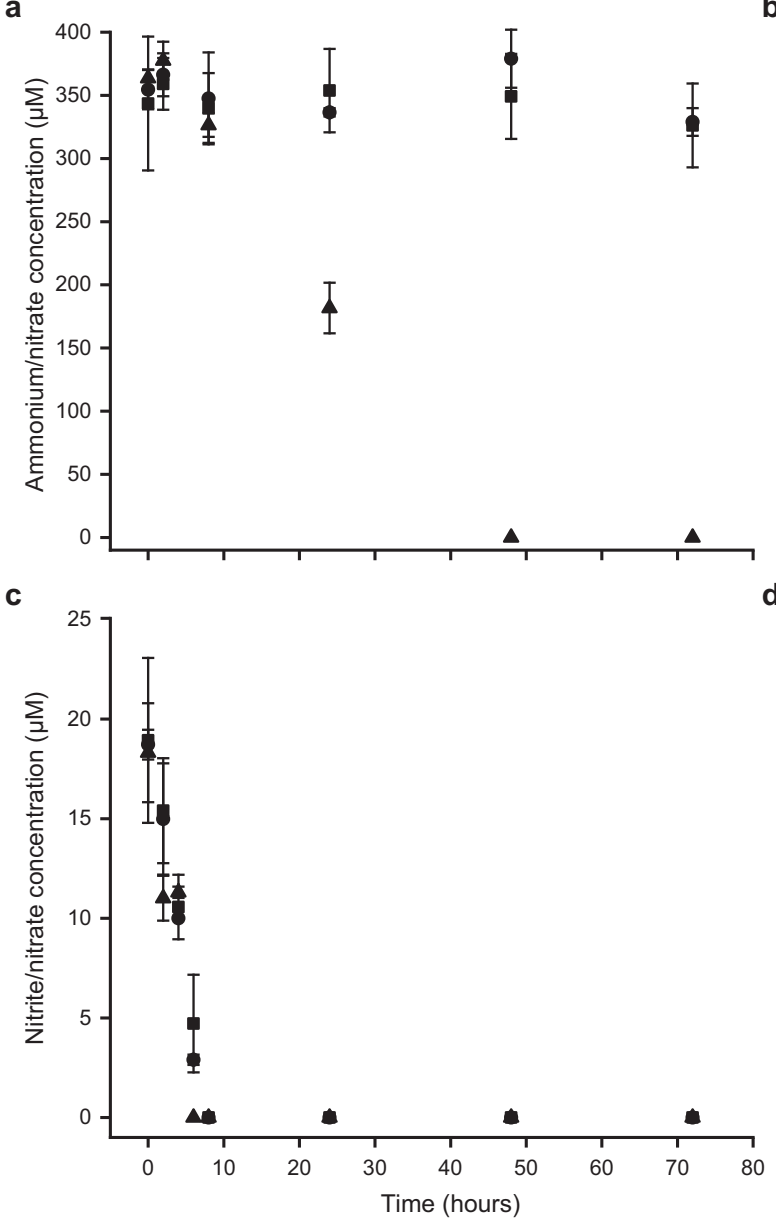

b
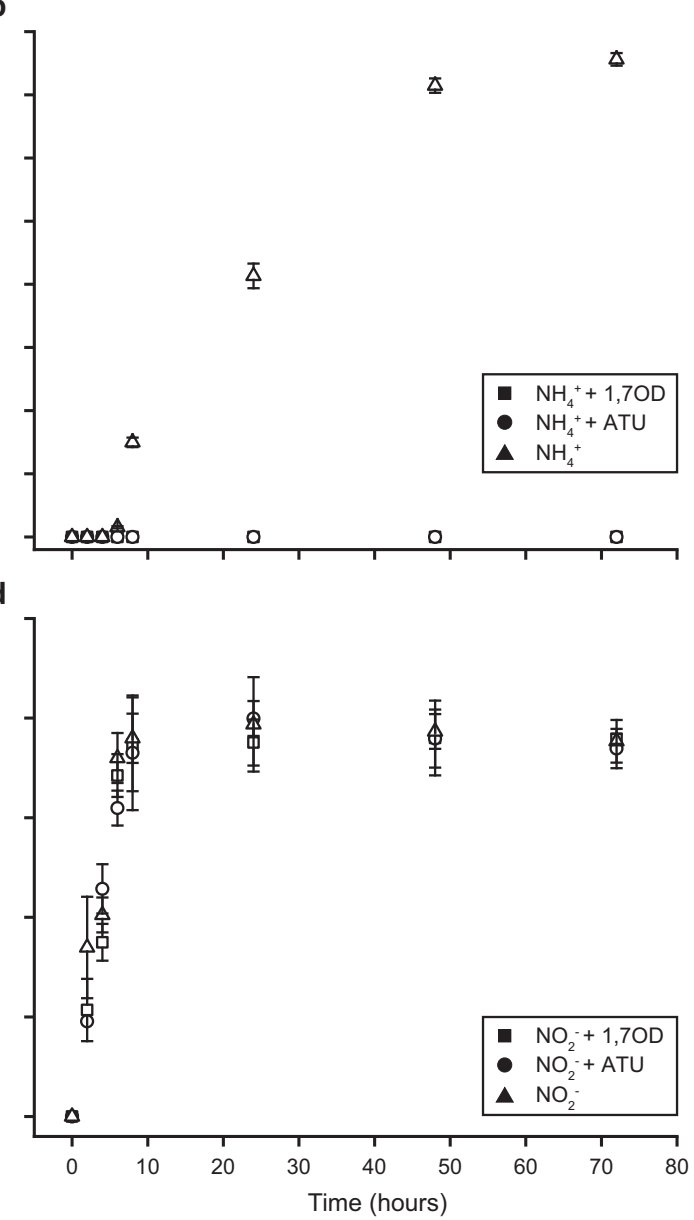

Fig. 1 Inhibition of ammonia oxidation activity of the comammox organism $\mathbf{C a}$. $\mathbf{N}$. kreftii by 1,70D. a Ammonium consumption and b nitrate production in the presence of $350 \mu \mathrm{M} \mathrm{NH}_{4}{ }^{+}$with and without the addition of $100 \mu \mathrm{M} 1,7 \mathrm{OD}$ or ATU. c Nitrite consumption and d nitrate production in the presence of $20 \mu \mathrm{M} \mathrm{NO}_{2}{ }^{-}$with and without the addition of $100 \mu \mathrm{M} \mathrm{1,70D}$ or ATU. Concentrations of the substrates ammonium (a) and nitrite (c) are indicated by solid, produced nitrate (b, d) by open symbols. Data points represent the mean of technical triplicates, error bars the standard deviations between three biological replicates.

pHMO and sHMO enzymes, which however was reported to use the pHMO for the oxidation of propane [41]; (vi) T. butanivorans, an alkane-oxidizing betaproteobacterium containing sHMO only [65]. Specific 1,70D-dependent fluorescent staining of all tested cultures indicated efficient activity-based labeling of these alkaneoxidizing bacteria, regardless of enzyme type and phylogenetic affiliation (Fig. 2 and Supplementary Figs. S1 and S2). Still, the full range of hydrocarbon-degrading monooxygenases targeted by this method remains to be determined.

\section{Correlation of the activity-based staining with the bacterial growth stage}

Laboratory cultivation conditions poorly reflect the conditions microorganisms encounter in natural and engineered ecosystems. In these environments, fluctuations in substrate and nutrient availability rarely permit the continuous growth of microorganisms, including AOB [104]. To investigate the potential effect of the bacterial growth stage on the efficiency of the activity-based staining, $N$. europaea and $N$. multiformis pure cultures were cultivated in the presence of 10 or $1 \mathrm{mM}$ ammonium, respectively, and ammonia-oxidizing activity and growth were monitored over 22 days. The cultures stoichiometrically oxidized ammonium to nitrite (Fig. 3a, b) and biomass samples taken at different time points were subjected to the activity-based labeling protocol. For $N$. europaea, the obtained fluorescent signal intensity reached its maximum at the start of the exponential growth phase (day 7) and decreased when the culture entered early stationary phase, but maintained a low signal intensity (35\% compared to the maximum intensity) even in late stationary phase (Fig. 3c). Throughout all growth stages of the culture a high percentage ( $>90 \%)$ of cells was stained, with a small decrease (16\%) in staining efficiency observed only in late stationary phase (Fig. 3c). In the case of $N$. multiformis, the activity-based staining-derived fluorescent signal reached its maximum intensity at the end of the exponential growth phase (day 18) and decreased by $30 \%$ once the culture reached its stationary phase (Fig. $3 \mathrm{~d}$ ). Also for this AOB a staining efficiency $>86 \%$ was observed throughout all growth stages (Fig. 3d).

Subcellular localization of active AMO and p/sMMO enzymes $\mathrm{AMO}$ and $\mathrm{pMMO}$ like all enzymes within the CuMMO protein family are known to be membrane-integral [42], with their active center on the periplasmic face of the cytoplasmic membrane or the ICM systems $[105,106]$. In contrast, the sMMO is a soluble cytoplasmic enzyme [37]. To confirm these subcellular localizations, pure cultures of the canonical AOB $N$. europaea, the comammox bacterium $N$. inopinata, the type lb methanotroph $M$. oryzae (containing only $\mathrm{pMMO}$ ), and the methanotroph $M$. tundrae (containing only SMMO) were subjected to the activity-based staining protocol in combination with $\mathrm{FISH}$ and subsequent deconvolution fluorescence microscopy (Fig. 4). In N. europaea, the AMO label was observed mainly in a thick layer surrounding 

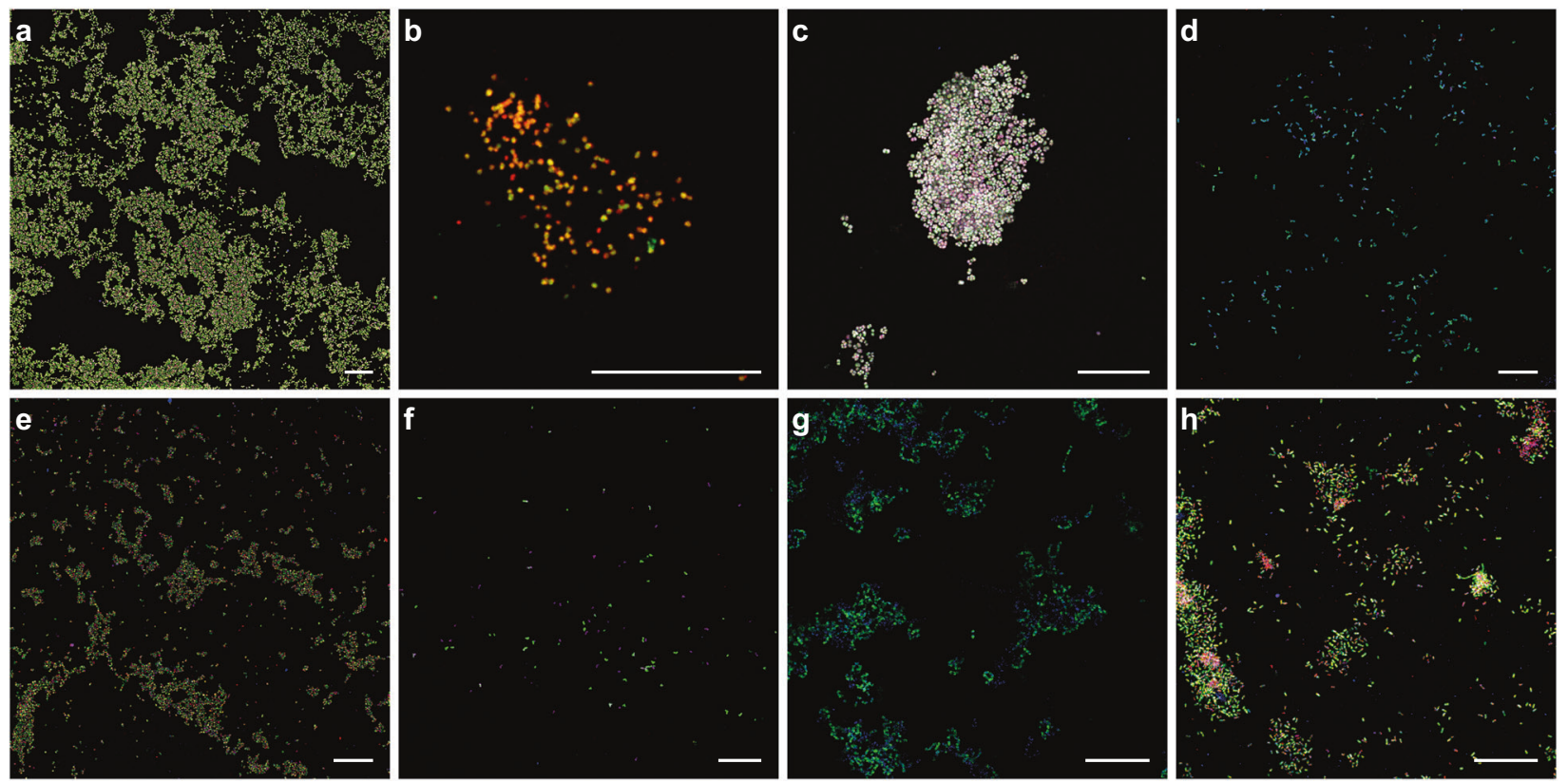

Fig. 2 Activity-based fluorescent labeling of catalytically active ammonia-, methane-, and other alkane-oxidizing microorganisms. Fluorescent micrographs of 1,70D pre-incubated cells of a Nitrosomonas europaea, b Nitrospira inopinata, c Methylotetracoccus oryzae, d Methylosinus sporium M29, e Methylacidiphilum fumariolicum SolV, f Methylocella tundrae, g Rhodococcus sp. strain ZPP, and h Thauera butanivorans. Cells were stained with the activity-based labeling protocol (shown in green) and FISH probes specific for a AOB (Nso190, Neu653, Nso1225; red), b Nitrospira (Ntspa662, Ntspa712; red), c, f Gammaproteobacteria (Gam42a; red), d Alphaproteobacteria (Alf0001b, Alf0968; red), e Verrucomicrobia (EUB338Ill; red), h Betaproteobacteria (Bet42a; red), and a-f, h) all bacteria (EUB338mix; blue). Cells in g were counterstained with DAPI (blue). Scale bars correspond to $20 \mu \mathrm{m}$.

the cytoplasm (Fig. 4a), coinciding with the localization of the cytoplasmic membrane and the peripheral organization of the ICM system in Nitrosomonas [106-108]. In N. inopinata the small cell size $(\leq 1 \mu \mathrm{m})$ precluded an unambiguous localization of the derived fluorescent signal, but the intense activity-based labeling indicated the presence of high amounts of active AMO enzymes along the cell membranes (Fig. 4b). In M. oryzae the ICMs are arranged as stacks of vesicular discs located in the central cytoplasm [105], which agrees with the observed strong pMMO fluorescent staining on apparently organized structures within the cytoplasm (Fig. 4c). As expected, in M. tundrae the sMMO labeling was distributed throughout the cytoplasm of the cell (Fig. 4d); however, there was only partly overlap with the $16 \mathrm{~S}$ rRNA-derived signal, indicating that methane oxidation and translation occur in discrete locations within the cell.

In order to examine the localization of active $\mathrm{AMO}$ and $\mathrm{PMMO}$ enzymes at higher resolution, cells of $N$. europaea and $M$. oryzae were subjected to a modified version of the activity-based staining protocol. Here, the CuAAC reaction was used to biotinylate the $\mathrm{AMO} / \mathrm{pMMO}$ enzymes within active bacterial cells, with subsequent immunogold labeling and transmission electron microscopy, thus providing high-resolution information on the subcellular localization of the complexes (Fig. 5). In N. europaea, $74 \%$ of the immunogold labeling indicated a membrane localization of the AMO complex, and the majority of these gold particles (49\%) appeared to face the periplasmic space, with only $13 \%$ oriented toward the cytoplasm (Fig. 5a, b and Supplementary Table S3). In accordance to the proposed localization of the alkynylation site in the MOB Methylococcus capsulatus [52], 61\% of gold labeling in $M$. oryzae depicted a membrane localization of the pMMO active center, with $71 \%$ of these membrane-associated labels on the periplasmic side of the membrane (Fig. $5 \mathrm{~d}$, e and Supplementary Table S3). In both cases, gold particles were seldomly found in the cytoplasm or outside the cell, indicating the high specificity of the labeling protocol (Fig. 5 and Supplementary Table S3).

\section{In situ activity-based fluorescent labeling of ammonia} oxidizers in nitrifying enrichments

The ability of the activity-based labeling protocol to stain ammonia oxidizers present in mixed microbial communities was assessed in different nitrifying enrichment cultures. We tested our newly developed protocol on the previously described enrichment culture of $\mathrm{Ca}$. N. nitrosa and $\mathrm{Ca}$. N. nitrificans [14], and a nitrifying enrichment containing canonical $A O B$, and both comammox and canonical Nitrospira, in addition to a range of putatively heterotrophic organisms. Furthermore, we demonstrated that a combination of the activity-based labeling protocol with $16 \mathrm{~S}$ rRNA-targeted FISH allowed the simultaneous phylogenetic identification of the ammonia-oxidizing bacterial populations in all samples (Fig. 6).

The activity-based labeling protocol was able to stain both types of $A O B$ present in these enrichment cultures. Fluorescent labeling of the AMO of comammox bacteria was observed both in the culture containing $\mathrm{Ca}$. N. nitrosa and $\mathrm{Ca}$. N. nitrificans (Fig. 6a), and in the nitrifying enrichment culture (Fig. $6 \mathrm{c}$ ). In the latter, canonical betaproteobacterial AOB were also efficiently doublestained by the activity-based labeling protocol and FISH, while some Nitrospira were detected by FISH only, presumably corresponding to canonical Nitrospira present in this sample.

\section{Targeted metagenomics}

In combination with FACS, our activity-based labeling protocol could be employed for targeted metagenomics of CuMMO and sHMO-containing bacteria present in complex environmental samples. To prove the feasibility of this approach, we tested the protocol on two samples of different complexity, a nitrifying enrichment culture and activated sludge from a full-scale WWTP.

Successive to the application of the activity-based labeling protocol and floc disruption using mild sonication, biomass of the nitrifying enrichment culture was used for FACS (Supplementary Fig. S3), with pooled collection of all positive sorting events. This fraction was subjected to DNA extraction and metagenomic 
a

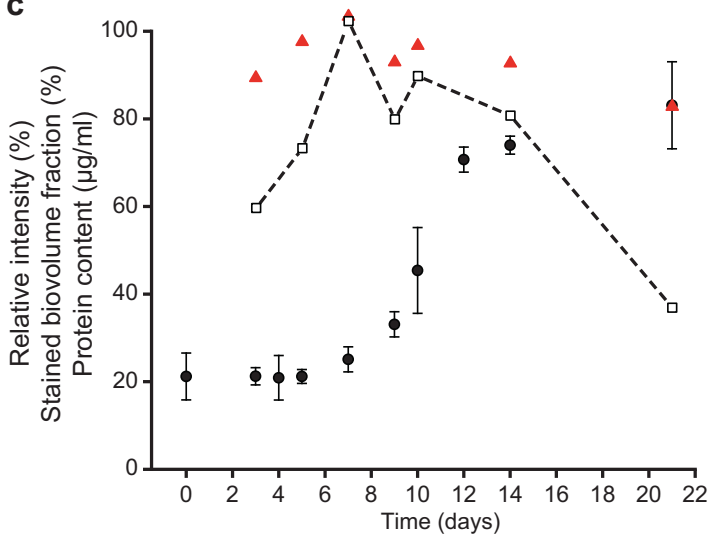

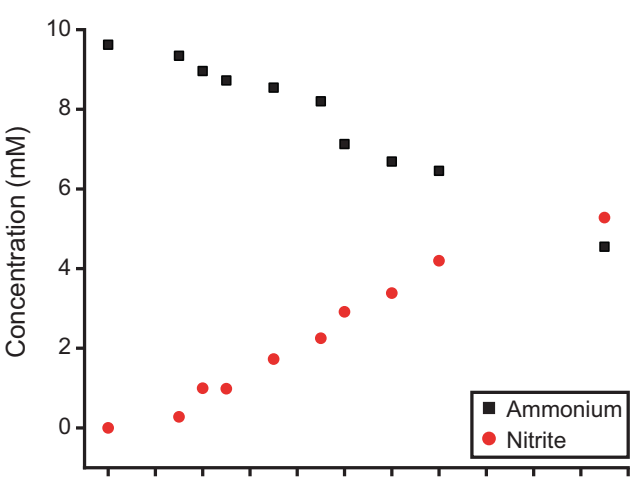

C

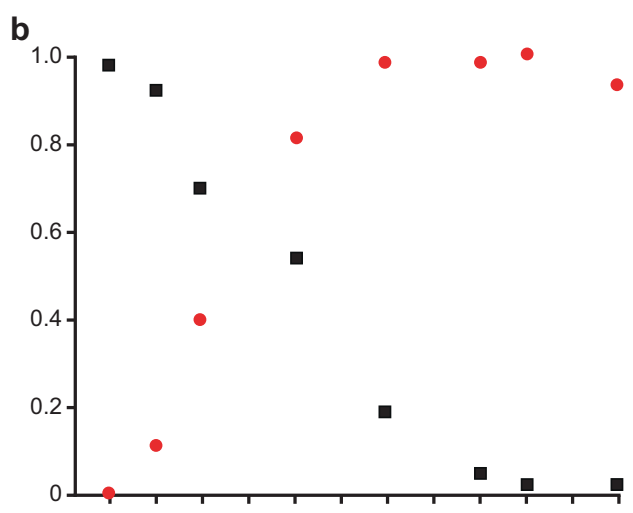

d

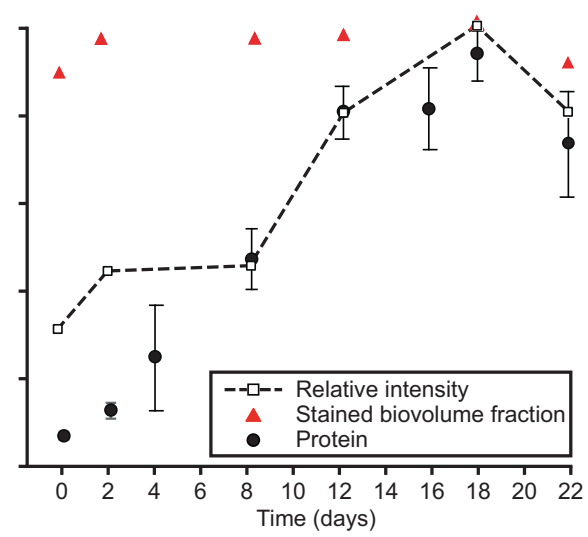

Fig. 3 Correlation of the activity-based fluorescent labeling signal intensity and staining efficiency with the growth stage of ammoniaoxidizing bacteria. Ammonium consumption and nitrite production activity by a Nitrosomonas europaea and $\mathbf{b}$ Nitrosospira multiformis during the incubation period. Growth of $\mathbf{c} N$. europaea and $\mathbf{d} N$. multiformis as indicated by protein content, and its relation to the relative fluorescent signal intensity observed, as well as the biovolume fraction of the culture stained by the activity-based labeling protocol. Bacterial growth data are fitted to a Hill equation. Error bars represent standard deviation of technical triplicates of protein measurements.

sequencing, along with an untreated biomass sample and a range of controls to test for potential biases in the protocol (Supplementary Table S2). To demonstrate the efficacy of the labeling and sorting protocol in a manner unbiased by metagenomic binning approaches and different sequencing depths, we used metagenomic read mapping to assembled marker genes for specific functions, i.e., CuMMO genes, and microbial taxa, i.e., RNA polymerase subunit $\mathrm{B}(r p o B)$, to detect and differentiate all members of the microbial community. In total, ten CuMMO genes were identified in the co-assembled contigs retrieved from the nitrifying enrichment: three were highly similar to AMO genes of Nitrosomonas and two of comammox Nitrospira (>96\% amino acid identity), while the remaining five were most similar to unresolved genes within proteobacterial lineages not known to oxidize ammonia (Supplementary Fig. S4). These latter genes clustered with putative pHMOs $[42,109]$ and became undetectable in the sorted sample. A total of 49 rроB genes were recovered and analyzed, including two affiliated with Nitrosomonas ( $>90 \%$ amino acid identity), two with comammox Nitrospira (>97\%), six with canonical Nitrospira (>97\%), and two with Candidatus Macondimonas diazotrophica (>90\%), a known hydrocarbon consumer that encodes a pHMO [110]. No rpoB genes affiliated with MOB or other lineages known to encode a CuMMO were apparent. For both marker genes belonging to the dominant ammonia oxidizers in the bioreactor, the normalized read abundances increased in the sorted samples compared to the unsorted samples: Nitrosomonas and comammox Nitrospira amoA increased 41- and 13-fold, and their $r p o B 86$ and 12-fold, respectively (Fig. 7). This increase in abundance was decoupled from the total number of reads per sample recruited to the co-assembly, indicating the changes were not due to variations in sequencing depth (Fig. 7). Finally, the abundance of non-ammonia-oxidizing community members' rpo $B$ genes decreased from $93.6 \%$ of the total normalized read abundance to $12.5 \%$, indicating a strong de-enrichment of the microbial community members lacking AMO (Fig. 7). Therefore, the activity-based fluorescent labeling coupled to cell sorting successfully and specifically enriched the ammonia-oxidizing microorganisms present in the nitrifying enrichment bioreactor.

However, the activity-based labeling and sorting approach did reveal biases and limitations. First, there appeared to be enrichment of nitrifying bacteria due to treatment steps prior to sorting, particularly among the sonicated and ethanol-treated samples, but also by the CuACC reaction itself (Fig. 7). These effects may be due the tendency of nitrifying bacteria to grow as microcolonies within biomass aggregates, which may protect them from the effects of sonication, ethanol fixation, and the CuAAC reaction [92-94]. Second, the apparent most abundant ammonia oxidizer after activity-based labeling and cell sorting depended on the marker gene analyzed. Based on AMO gene abundance increases, a Nitrosomonas-like canonical AOB appeared to be more highly enriched, while this was a comammox Nitrospira according to $r p o B$ abundance. The slopes of linear correlations $\left(r^{2}>0.95\right.$; Supplementary Fig. S5) of the most abundant CuMMO and corresponding rpo $B$ genes support genomic evidence that this discrepancy may be an effect of gene copy number, as Nitrosomonas species typically encode three copies of the AMO gene but comammox Nitrospira only one [111]. As a side note, one putative bacterial hydrocarbon degrader- 

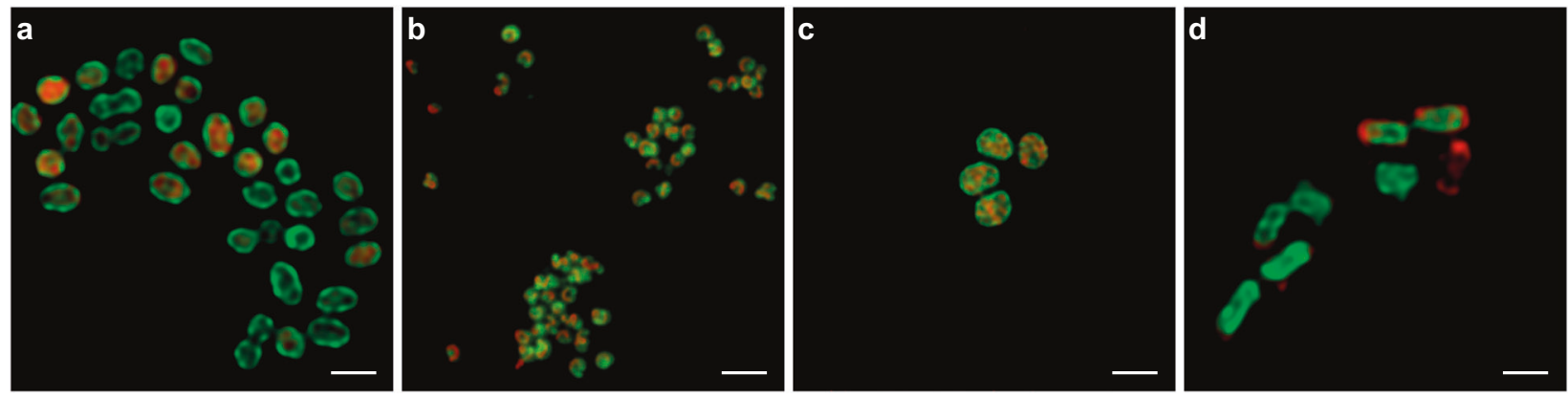

Fig. 4 Subcellular localization of the active enzymes using activity-based fluorescent labeling signal and deconvolution confocal microscopy. Cells of a Nitrosomonas europaea, b Nitrospira inopinata, c Methylotetracoccus oryzae, and d Methylocella tundrae are stained with the fluorescent activity-based labeling protocol (shown in green) and specific FISH probes (shown in red): a AOB (Nso190, Neuo653, Nso1225), b Nitrospira (Ntspa662, Ntspa712), c Gammaproteobacteria (Gam42a), and d Alphaproteobacteria (Alf0001b, Alf0968). The scale bars correspond to $1 \mu \mathrm{m}$.

affiliated $r p o B$ was strongly correlated $\left(r^{2}>0.9\right)$ to two different, similarly abundant putative pHMO genes across all samples, suggesting these microorganism may use distinct CuMMO enzymes able to activate a variety of alkanes (Supplementary Fig. S5); however, strong de-enrichment of this putative alkane oxidizer during sorting indicated that these pHMOs were not active under the incubation conditions of the enrichment culture (Fig. 7). Third, the larger increases in abundance of both Nitrosomonas marker genes compared to comammox Nitrospira suggest that the technique may slightly favor Nitrosomonas-like $A O B$. Fourth, some $A M O$ genes and $r p o B$ affiliated with different ammonia oxidizers were either poorly enriched or even deenriched, which makes it tempting to speculate that the physiology of these seemingly related populations differ in vivo to the extent that some do not express AMO (Fig. 7). While it is important to acknowledge and account for these caveats, metagenomic recovery and abundance estimations clearly showed effective enrichment of ammonia-oxidizing microorganisms by the activity-based labeling protocol coupled to FACS.

When our activity-based labeling protocol was applied to activated sludge sampled at a municipal WWTP, bacterial ammonia-oxidizing populations were also successfully enriched. Co-assembly of the untreated sludge biomass, controls, and sorted samples yielded two Nitrosomonas bins and two Nitrospira bins (Supplementary Table S4). The two Nitrosomonas bins increased 8and 53-fold in mean normalized read abundance compared to the untreated biomass, while the Nitrospira bins increased 4-fold (Fig. 8 and Supplementary Fig. S6). These four bins accounted for $1.1 \%$ of the normalized reads in the original sample and less than $20 \%$ of their contigs were detected (covered bases $>50 \%$ ), but after activity-based labeling and sorting these bins constituted $11.5 \%$ of the normalized reads, suggesting that sorting was able to aid in the recovery of otherwise inaccessible genomes of AOB. Though not automatically binned, four partial and two full-length Nitrosomonas-affiliated AMO genes were assembled, as well as one putative pHMO gene affiliated with Proteobacteria (Supplementary Fig. S4). No comammox Nitrospira-related AMO gene was detected in the assembly, indicating that the enriched Nitrospira bins belonged to canonical nitrite-oxidizing species that were sorted together with their ammonia-oxidizing partners due to their close physical interaction [112]. The two full-length Nitrosomonas AMO genes showed similar patterns as those in the nitrifying enrichment bioreactor because they were approximately 3.8-fold more abundant in the sorted metagenome than the likely bin. Assembly and binning of the sorted sample alone led to the recovery of a high-quality Nitrosomonas MAG ( $>99 \%$ complete, $<1 \%$ contaminated), enabling the assignment of one of the Nitrosomonas AMO genes to this MAG (Supplementary Fig. S4). Contrastingly, the low abundance and lack of matching abundance patterns with any bin prevented assignment of the putative
pHMO. Thus, despite the challenges of assembling and binning closely related strains of ammonia oxidizers, the ABPP-based protocol in combination with targeted cell sorting was clearly capable of enriching low-abundant ammonia oxidizers from complex activated sludge samples.

Surprisingly, over $72 \%$ normalized reads obtained for the sorted activated sludge sample were recruited to bins affiliated with putative glycogen-accumulating organisms (GAOs) of the family Competibacteraceae (Supplementary Table S4). In addition, all eight automated bins increased in mean normalized read abundance approximately 4.7- to 18.5-fold, suggesting that they were also enriched by the activity-based labeling and sorting. Their high abundance in the sorted sample enabled the recovery of four medium-quality MAGs $(61-96 \%$ complete, $<10 \%$ contaminated) from an assembly of the sorted sample alone, which represent three distinct genera (Supplementary Table S5). No genes encoding known members of the CuMMO or sHMO protein families were detected in these bins or MAGs, suggesting that the apparent labeling (Supplementary Fig. S7) and enrichment by sorting was due to other factors. Instead, all but one of the Competibacteraceae MAGs encoded nitrogenases, which is an enzyme known to interact with alkynes and may explain the unexpected enrichment [113].

To test for potential labeling of the nitrogenase complex, we incubated pure cultures of the diazotroph Krypidia spormannii FAVT5 [66] and the methanotroph $M$. oryzae [64] under nitrogenfixing conditions. Subsequently, both cultures were subjected to the activity-based labeling protocol. As $M$. oryzae contains a pMMO, staining of these cells was observed, but compared to a culture grown in the presence of nitrate as $\mathrm{N}$-source in the medium, the staining intensity was slightly reduced (Supplementary Fig. S2). For $K$. spormannii FAVT5, the amount of signal observed after the CuAAC reaction was just above background, corresponding to approximately $12 \%$ and $25 \%$ of the signal obtained from active $N$. europaea and $M$. tundrae cultures, respectively (Supplementary Fig. S2). Together, these results indicate that the presence of an active nitrogenase does minimally contribute to the activity-based labeling, making the reason for the observed staining of the Competibacteraceae-affiliated MAGs unclear. It thus remains to be discovered which enzymes apparently are able to react with 1,70D and what their natural function is in the metabolism of these Competibacteraceae species.

\section{DISCUSSION}

ABPP-based protocols have been successfully employed for the study of many microbial protein families, such as proteases, kinases, hydrolases, and glycosidases [20, 29]. However to date, they have rarely been applied for the in situ labeling of whole cells within microbial communities, due to redundant, poorly resolved, 

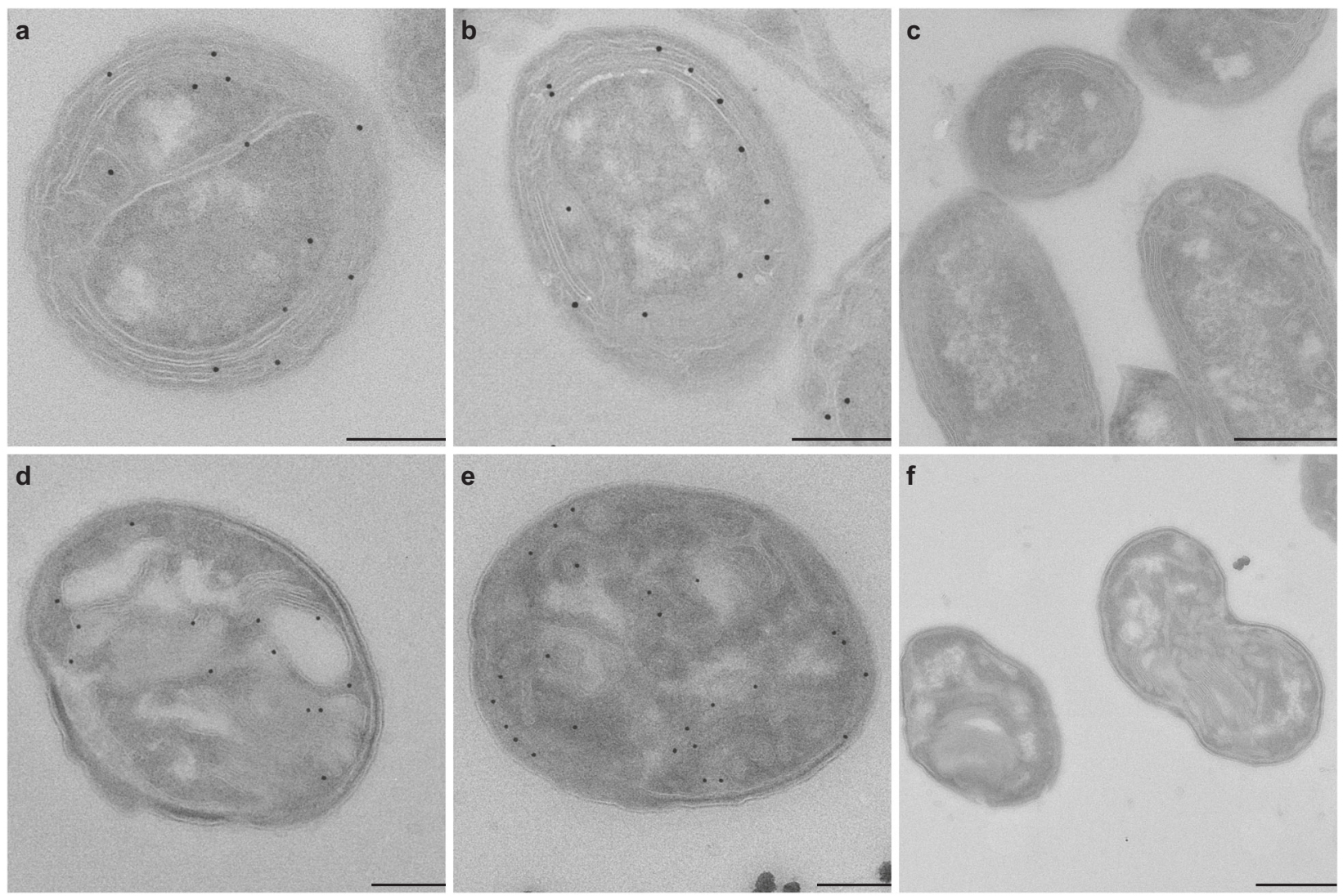

Fig. 5 Immunogold activity-based labeling of active enzymes using transmission electron microscopy. Electron micrographs showing $\mathbf{a}-\mathbf{c}$ Nitrosomonas europaea and $\mathbf{d}-\mathbf{f}$ Methylotetracoccus oryzae cells pre-incubated (a, b, d, e) with and (c, $\mathbf{f}$ ) without 1,70D (control). Scale bars correspond to $0.5 \mu \mathrm{m}$.
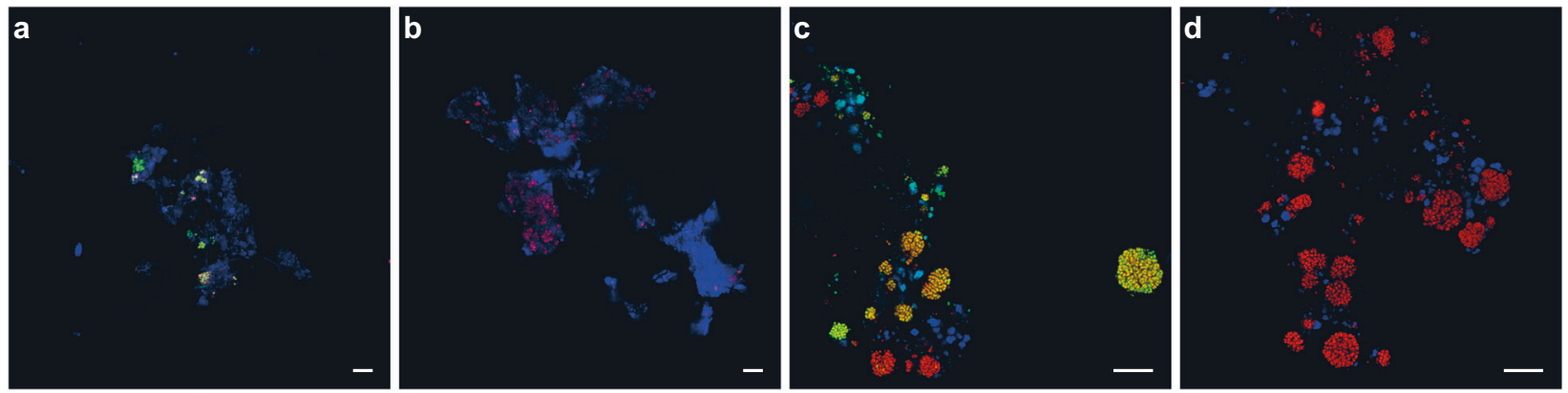

Fig. 6 Activity-based fluorescent labeling of catalytically active ammonia-oxidizing bacteria present in complex microbial communities. Fluorescent micrographs showing $\mathbf{a}$, $\mathbf{b}$ a culture containing $\mathrm{Ca}$. N. nitrosa and $\mathrm{Ca}$. N. nitrificans and $\mathbf{c}$, $\mathbf{d}$ a nitrifying enrichment culture preincubated $(\mathbf{a}, \mathbf{c})$ with and $(\mathbf{b}, \mathbf{d})$ without 1,7OD (control). Cells are stained with the activity-based labeling protocol (shown in green) and FISH probes specific for Nitrospira (Ntspa662, Ntspa712; shown a, b in red and c, d in blue), c, d ammonia-oxidizing bacteria (Nso190, NEU653, Nso1225; in red), and a, b all bacteria (EUB338mix; in blue). Scale bars correspond to $10 \mu \mathrm{m}$.

or unclearly connected taxa or functions [114]. In situ use of ABPPbased protocols poses major challenges for employing the CuAAC reaction, for example probe uptake, and labeling specificity and efficiency [115]. The activity-based labeling protocol developed in this study overcomes these challenges and allows the fluorescent visualization of specific catalytically active enzymes in situ. The protocol makes use of the diyne 1,70D, which serves as a bifunctional enzyme probe for the specific detection of CuMMO and sHMO enzymes in diverse ammonia- and alkane-oxidizing bacteria. The feasibility of this approach was previously demonstrated in vitro for the AMO of canonical AOB [31], but had not been tested for labeling whole cells, phylogenetically distinct ammonia-oxidizing microorganisms, or evolutionarily or functionally related enzymes. Generally, $n$-terminal and sub-terminal alkynes are known mechanism-based inactivators of AMO $[53,54]$ and $\mathrm{p} / \mathrm{sMMO}$ enzymes $[51,56]$. Here, we revealed that $1,7 \mathrm{OD}$ also inhibits ammonia oxidation in comammox Nitrospira (Fig. 1) as efficiently as was reported for canonical AOB [31]. Furthermore, strong labeling of different methane- and other alkane-oxidizing bacteria harboring diverse $\mathrm{pHMO}$ or sHMO enzymes (Fig. 2) indicated that methanotrophs and alkaneoxidizing bacteria can also be inhibited by this diyne.

Application of the in situ activity-based protocol on pure and enrichment cultures of different canonical AOB, comammox Nitrospira, and alkane oxidizers resulted in efficient, mechanismbased fluorescent labeling (Fig. 2 and Supplementary Fig. S2). This directly conveyed information on the functional potential of the detected microorganisms, in contrast to conventional in situ 
a

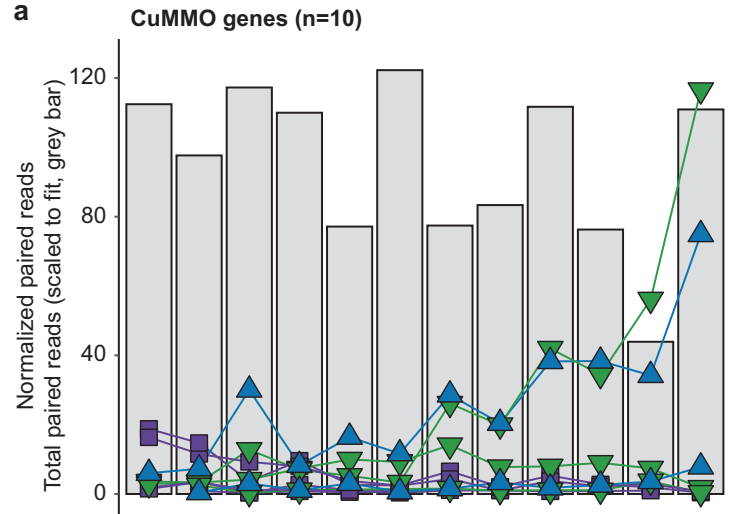

b

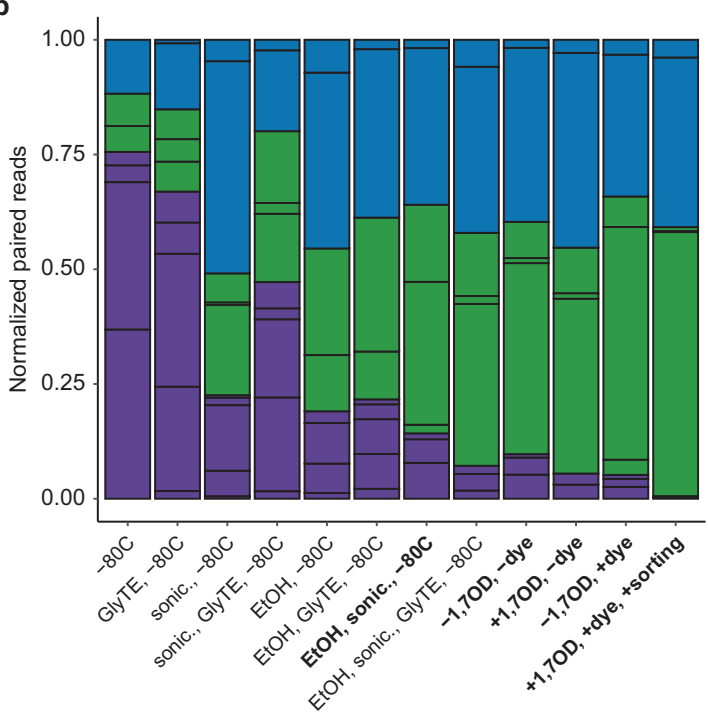

Marker affiliations:
C $\operatorname{rpoB}(\mathrm{n}=49)$

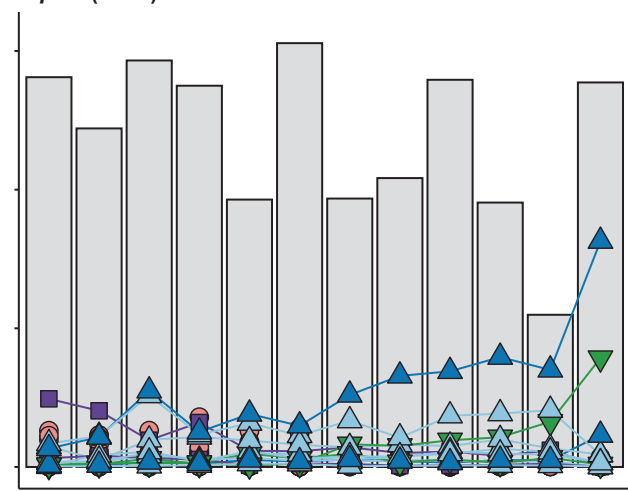

d

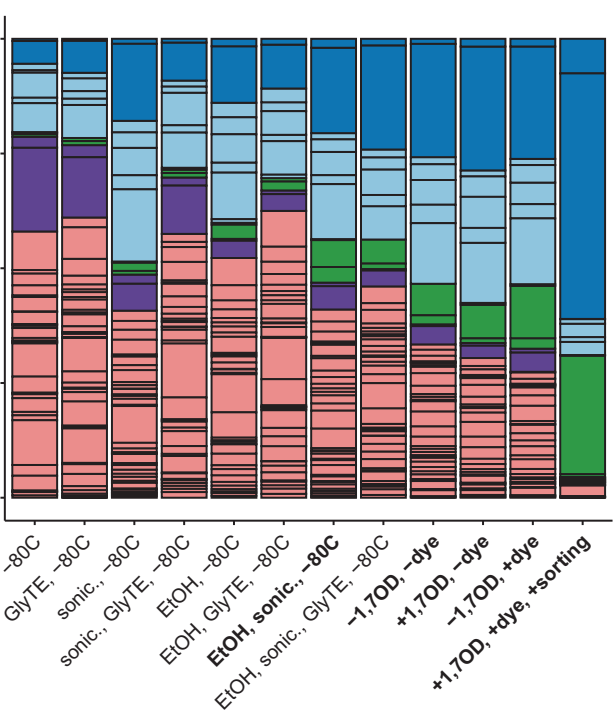

Fig. 7 Targeted metagenomics of CuMMO-containing bacteria. Normalized read abundances of a, $\mathbf{b}$ CuMMO genes and $\mathbf{c}$, $\mathbf{d}$ rpoB in the nitrifying enrichment culture of untreated biomass, controls, and sorted biomass for the activity-based labeling protocol (see Supplementary Table S2 for details). Gene abundances were calculated by normalizing paired read counts by gene length and million reads mapped to the co-assembly. The total number of reads mapped to the co-assembly are indicated as gray bars scaled to fit in the same range as the normalized abundances. Colors denote the taxonomic affiliation of the marker genes. The same genes for the taxa of interest (Nitrosomonas, comammox Nitrospira, and putative alkane oxidizers) are emphasized and connected with lines. b, $\mathbf{d}$ show the same data as (a, c) but as proportion of the total normalized read abundance for the respective gene in the metagenomic sample, with each individual colored box representing the relative abundance of a single gene from the co-assembly. The final treatment preceding the CuAAC reaction, the CuAAC controls for the influence of 1,7OD inactivation and dye addition, and the final sorted sample are indicated in bold.

detection methods like FISH. Still, in most natural and engineered systems, microorganisms rarely encounter optimal growth conditions and might co-exist in heterogeneous growth stages. Previous studies have identified preservation of basal activity levels of ammonia- and methane-oxidizing microorganisms as a general adaptation to cope with alternating conditions and fluctuating substrate supply [104, 116], which therefore maintain a basal CuMMO content. Quantification of the activity-based fluorescent labeling signals in N. europaea and $N$. multiformis pure cultures showed strong correlations between signal intensity and growth state (Fig. 3). As expected, the strongest signals were observed during the exponential growth phase, corresponding to a high AMO content of the cells. Lower but detectable labeling was still observed even in the late stationary phase of the cultures, demonstrating that basal AMO contents are also maintained in non-replicating cells. Thus, although the intensity of the activitybased labeling might vary, the approach is suitable for the detection of ammonia- and methane-oxidizing cells in different growth stages. However, exposure to their specific substrate may be required to stimulate expression of the target enzymes by metabolically versatile microorganisms for this activity-based labeling protocol, as indicated by the lack of labeling of some Nitrosomonas species and the pHMO-containing putative alkane oxidizers detected in the nitrifying enrichment culture (Fig. 7).

Unfortunately, it was not possible to label the archaeal AMO using $1,70 D$ as bifunctional enzyme probe (Supplementary Fig. S2i), consistent with the low sensitivity of AOA to longerchain length $\left(>C_{5}\right)$ alkynes and their reversible inhibition by 1-octyne [54]. Nevertheless, these microorganisms are sensitive to inactivation by $n$-alkynes with short carbon backbones $\left(\leq C_{5}\right)$ [31], and thus substituting 1,7OD with an alkyne with a smaller chain length might permit labeling of archaeal AMO. However, the fact that diynes smaller than 1,5-hexadiyne are extremely reactive and difficult to obtain makes modification of this method for the detection of AOA challenging.

Using high-resolution deconvolution laser scanning microscopy as well as electron microscopy, the activity-based labeling protocol permitted visualization of the subcellular localization of the $\mathrm{AMO}$ and $\mathrm{p} / \mathrm{sMMO}$ enzymes in $\mathrm{AOB}$ and MOB (Figs. 4 and 5). In accordance with previous studies [63, 107, 108], the AMO and 


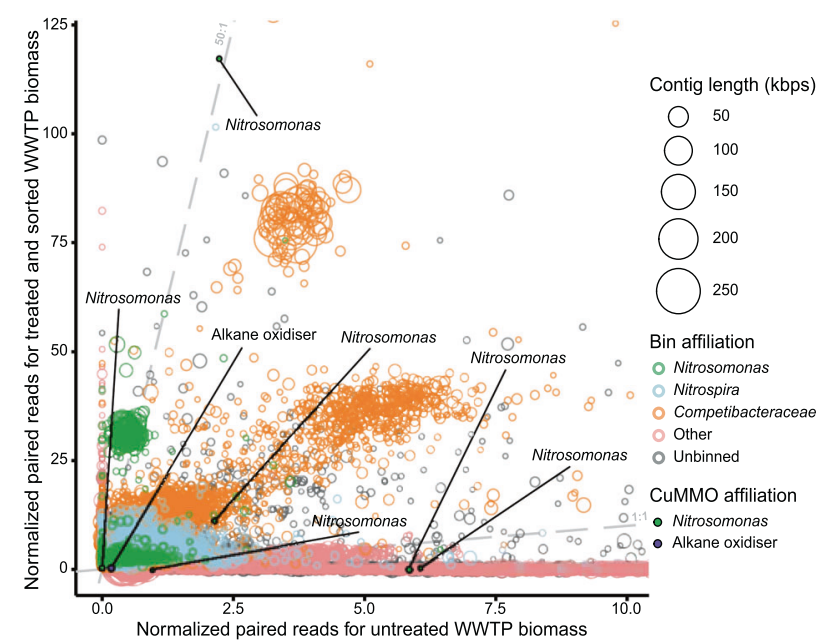

Fig. 8 Differential coverage plot showing the abundance of contigs in the municipal WWTP untreated activated sludge sample in comparison to the activity-based labeled and sorted sample. Contig abundances were calculated by normalizing paired read counts by gene length and million reads mapped to the assembly. Each circle represents an assembled contig $\geq 1500 \mathrm{bps,}$ indicated by circle size. Contigs assigned to the automated bins of taxa of interest are highlighted using specific colors. Contigs with genes encoding members of the CuMMO protein family are emphasized with fill circles and text. For added clarity, dashed lines were drawn with a slope of 1 and 50 to guide estimations of the differences in normalized abundances between the samples. The plot is zoomed in on the region containing $>80 \%$ of all data, the full version is provided as Supplementary Fig. S6.

pMMO-derived fluorescent signals were mostly localized along the cytoplasmic membrane and on the ICM stacks in N. europaea and $M$. oryzae, respectively, whereas a cytoplasmic localization of the sMMO-derived signal was observed for $M$. tundrae. Furthermore, the immunogold labeling data obtained in this study suggested that the alkynylation sites for both the AMO and PMMO complexes were found on the periplasmic face of the ICMs. This congruence with expected enzyme localizations further verified the suitability of the activity-based protocol for the specific labeling of CuMMO enzymes, which opens up possibilities for studying differences in enzyme distribution along intracellular structures in $A O B$ and alkane-oxidizing microorganisms with divergent cell morphologies. Although in theory direct tagging of the CuMMO using azide-functionalized gold nanoparticles could be employed in future enzyme localization studies, the biotinylation of these enzymes via the activity-based protocol as tested here can also provide a new methodology for the profiling or affinity purification to study various CuMMO and sHMO enzymes.

Generally, nitrifiers and methane oxidizers are notoriously difficult to cultivate due to their slow growth rates and susceptibility to contamination. Besides classical cultivation attempts, the identification of novel ammonia and methane oxidizers to date was achieved using cultivation-independent molecular techniques [117-119] and metagenomic sequencing $[2,13-17,120-123]$, which has been invaluable for providing insights into the distribution and ecological importance of these functional groups. Still, due to the complexity of natural ecosystems, metagenomic sequencing frequently fails to recover sufficient data to reconstruct genomes of low-abundance organisms, and linking novel sequence types detected in metagenomic or PCR-derived datasets to a specific phylotype often is a highly challenging and time-consuming task. In addition, PCR primers will mostly fail to amplify novel and divergent sequences, thereby hindering the detection of species with novel metabolic capabilities, as was showcased by the recent discovery of complete nitrification within members of the genus Nitrospira $[13,14]$. Thus, there is a pressing need for simple detection methods that do not require a priori knowledge on the identity of microorganism. The here-developed activity-based labeling protocol can be combined with FISH to directly link the function of a population to its identity in situ, which could help to identify novel microorganisms capable of utilizing ammonia, methane, or other short-chain alkanes. Along these lines, while amoA-targeting PCRbased methods by now have successfully been employed to detect the presence and abundance of comammox Nitrospira in complex microbial communities or environmental samples $[117,124,125]$, it remains challenging to phylogenetically differentiate canonical and comammox Nitrospira. In combination with FISH, the activity-based staining method described here overcomes this limitation and enables an reliable differentiation of comammox and canonical Nitrospira in mixed microbial communities (Fig. 6).

Furthermore, we combined the activity-based labeling protocol with cell sorting and subsequent metagenomic sequencing. Application of this protocol on a nitrifying bioreactor enrichment culture successfully enriched the Nitrosomonas-like betaproteobacterial $\mathrm{AOB}$ and comammox Nitrospira (Fig. 7). While the physical and chemical treatments of the biomass during the activity-based protocol introduced biases resulting in a slight unspecific enrichment of target populations also in the controls, sorting was highly specific for these ammonia-oxidizing microorganisms (Fig. 7). As discussed above, the observed unspecific enrichment may be due to the tendency of these bacteria to physically associate in microcolonies [126, 127]. Regardless, the activity-based fluorescent labeling coupled to targeted cell sorting was sufficient for strong enrichment of multiple AOB from a mixed microbial community. When applied to activated sludge from a full-scale municipal WWTP, the activity-based labeling protocol with subsequent cell sorting resulted in strong enrichment of Nitrosomonas-like AOB (Fig. 8), which initially were at low abundance and constituted $<1 \%$ of normalized reads in this sample. The activity-based labeling followed by cell sorting enriched them over 50 -fold and even enabled recovery of a high-quality Nitrosomonas MAG. With deeper sequencing, or for samples with sufficient biomass with additional long-read sequencing, it will likely even be possible to recover circular genomes using this targeted metagenomics approach.

Surprisingly, several MAGs belonging to members of the Competibacteraceae family were enriched from the activated sludge sample after activity-based labeling and sorting. Described Competibacteraceae species are GAOs, which frequently are encountered in enhanced biological phosphorus removal systems. They compete with phosphate-accumulating organisms for resources under the cyclic anaerobic feast-aerobic famine regime and thus reduce the phosphate removal efficiency of these wastewater treatment systems [128]. Their primary metabolism includes synthesis and storage of glycogen and polyhydroxyalkanoates, but they are also capable of fermentation, denitrification, and nitrogen fixation [129]. However, these bacteria have never been implicated in the nitrification process or hydrocarbon degradation, and the MAGs recovered here lacked genes that are known to react with 1,70D during the activitybased labeling approach. Therefore, the reason for their enrichment (Fig. 8 and Supplementary Fig. S7) through the activitybased labeling protocol remains unclear and requires future research.

In conclusion, we present a novel activity-based protocol that enables the efficient in situ labeling of CuMMO and sHMOencoding bacteria, can be used to better link function and identity, and facilitates the targeted retrieval of high-quality and 
metabolically constrained genomes for microbes of low abundance. Although optimization of the protocol for difficult sample types that exhibit high background fluorescence will be necessary, the developed activity-based labeling protocol is a significant milestone for integrating phylogenetic and physiological information that can be adapted and applied to complex microbial communities. Coupling this novel method with other common techniques can help reveal the functional differences in biomass aggregates of phylogenetically indistinguishable lineages, as well as enable the recovery of new genomic representatives critical for engineered processes that account for only a small portion of the microbial community. Therefore, the future application of this activity-based labeling protocol will contribute greatly to an improved understanding of the microorganisms involved in important carbon and nitrogen cycle processes within a variety of ecosystems.

\section{DATA AVAILABILITY}

Sequencing data obtained in this study have been deposited in the National Center for Biotechnology Information (NCBI) database under BioProject accession numbers PRJNA691748 (nitrifying enrichment culture) and PRJNA691751 (activated sludge sample).

\section{REFERENCES}

1. Mobarry BK, Wagner M, Urbain V, Rittmann BE, Stahl DA. Phylogenetic probes for analyzing abundance and spatial organization of nitrifying bacteria. Appl Environ Microbiol. 1996;62:2156-62.

2. Dunfield PF, Yuryev A, Senin P, Smirnova AV, Stott MB, Hou S, et al. Methane oxidation by an extremely acidophilic bacterium of the phylum Verrucomicrobia. Nature. 2007;450:879-82.

3. Happel E, Bartl I, Voss M, Riemann L. Extensive nitrification and active ammonia oxidizers in two contrasting coastal systems of the Baltic Sea. Environ Microbiol. 2018;20:2913-26.

4. Lösekann T, Knittel K, Nadalig T, Fuchs B, Niemann H, Boetius A, et al. Diversity and abundance of aerobic and anaerobic methane oxidizers at the Haakon Mosby Mud Volcano, Barents Sea. Appl Environ Microbiol. 2007;73:3348-62.

5. Kolb $\mathrm{S}$. The quest for atmospheric methane oxidizers in forest soils. Environ Microbiol Rep. 2009;1:336-46.

6. Bellucci M, Curtis TP. Chapter eleven - ammonia-oxidizing bacteria in wastewater. In: Klotz MG, Stein LY, editors. Methods in enzymology. 496. San Diego, CA, USA: Academic Press; 2011. p. 269-86.

7. Lawson $C E$, Lücker S. Complete ammonia oxidation: an important control on nitrification in engineered ecosystems? Curr Opin Biotechnol. 2018;50:158-65.

8. Stein LY. Insights into the physiology of ammonia-oxidizing microorganisms. Curr Opin Chem Biol. 2019;49:9-15.

9. Lehtovirta-Morley LE. Ammonia oxidation: ecology, physiology, biochemistry and why they must all come together. FEMS Microbiol Lett. 2018;365:fny058.

10. Bédard $\mathrm{C}$, Knowles R. Physiology, biochemistry, and specific inhibitors of $\mathrm{CH}_{4}$, $\mathrm{NH}_{4}{ }^{+}$, and $\mathrm{CO}$ oxidation by methanotrophs and nitrifiers. Microbiological Rev. 1989;53:68-84.

11. Dalton H. Ammonia oxidation by the methane oxidising bacterium Methylococcus capsulatus strain Bath. Arch Microbiol. 1977;114:273-9.

12. Poret-Peterson AT, Graham JE, Gulledge J, Klotz MG. Transcription of nitrification genes by the methane-oxidizing bacterium, Methylococcus capsulatus strain Bath. ISME J. 2008:2:1213-20.

13. Daims H, Lebedeva EV, Pjevac P, Han P, Herbold C, Albertsen M, et al. Complete nitrification by Nitrospira bacteria. Nature. 2015;528:504-9.

14. van Kessel MA, Speth DR, Albertsen M, Nielsen PH, Op den Camp HJ, Kartal B, et al. Complete nitrification by a single microorganism. Nature. 2015;528:555-9.

15. Könneke M, Bernhard AE, de la Torre JR, Walker CB, Waterbury JB, Stahl DA. Isolation of an autotrophic ammonia-oxidizing marine archaeon. Nature. 2005;437:543-6.

16. Ettwig KF, Butler MK, Le Paslier D, Pelletier E, Mangenot S, Kuypers MMM, et al Nitrite-driven anaerobic methane oxidation by oxygenic bacteria. Nature. 2010:464:543-8.

17. Pol A, Heijmans K, Harhangi HR, Tedesco D, Jetten MSM, Op Den Camp HJM. Methanotrophy below $\mathrm{pH} 1$ by a new Verrucomicrobia species. Nature. 2007;450:874-8.

18. Smith GJ, Wrighton KC. Metagenomic approaches unearth methanotroph phylogenetic and metabolic diversity. Curr Issues Mol Biol. 2019;33:57-84.
19. Zandt $M H$, de Jong $A E E$, Slomp $C P$, Jetten MSM. The hunt for the most-wanted chemolithoautotrophic spookmicrobes. FEMS Microbiol Ecol. 2018;94:fiy064.

20. Sadler NC, Wright AT. Activity-based protein profiling of microbes. Curr Opin Chem Biol. 2015;24:139-44.

21. Speers $A E$, Cravatt BF. Profiling enzyme activities in vivo using click chemistry methods. Chem Biol. 2004;11:535-46.

22. Rostovtsev VV, Green LG, Fokin VV, Sharpless KB. A stepwise Huisgen cycloaddition process: copper(I)-catalyzed regioselective "ligation" of azides and terminal alkynes. Angew Chem Int Ed. 2002;41:2596-9.

23. Tornøe CW, Christensen C, Meldal M. Peptidotriazoles on solid phase: $[1,2,3]$ triazoles by regiospecific copper(I)-catalyzed 1,3-dipolar cycloadditions of terminal alkynes to azides. J Org Chem. 2002;67:3057-64.

24. Köhn M, Breinbauer R. The Staudinger ligation-a gift to chemical biology. Angew Chem Int Ed. 2004;43:3106-16.

25. Greenbaum D, Baruch A, Hayrapetian L, Darula Z, Burlingame A, Medzihradszky $\mathrm{KF}$, et al. Chemical approaches for functionally probing the proteome. Mol Cell Proteom. 2002;1:60-8.

26. Kocaoglu O, Calvo RA, Sham LT, Cozy LM, Lanning BR, Francis S, et al. Selective penicillin-binding protein imaging probes reveal substructure in bacterial cell division. ACS Chem Biol. 2012;7:1746-53.

27. Joyce JA, Baruch A, Chehade K, Meyer-Morse N, Giraudo E, Tsai F-Y, et al. Cathepsin cysteine proteases are effectors of invasive growth and angiogenesis during multistage tumorigenesis. Cancer Cell. 2004;5:443-53.

28. Yestrepsky BD, Kretz CA, Xu Y, Holmes A, Sun H, Ginsburg D, et al. Development of tag-free photoprobes for studies aimed at identifying the target of novel Group A Streptococcus antivirulence agents. Bioorg Medicinal Chem Lett. 2014;24:1538-44.

29. Wright AT, Cravatt BF. Chemical proteomic probes for profiling cytochrome p450 activities and drug interactions in vivo. Chem Biol. 2007;14:1043-51.

30. Saghatelian A, Jessani N, Joseph A, Humphrey M, Cravatt BF. Activity-based probes for the proteomic profiling of metalloproteases. Proc Natl Acad Sci USA. 2004;101:10000-5.

31. Bennett K, Sadler NC, Wright AT, Yeager C, Hyman MR. Activity-based protein profiling of ammonia monooxygenase in Nitrosomonas europaea. Appl Environ Microbiol. 2016;82:2270-9.

32. McTavish $\mathrm{H}$, Fuchs JA, Hooper AB. Sequence of the gene coding for ammonia monooxygenase in Nitrosomonas europaea. J Bacteriol. 1993;175:2436-44.

33. Pratscher J, Vollmers J, Wiegand S, Dumont MG, Kaster A-K. Unravelling the identity, metabolic potential and global biogeography of the atmospheric methane-oxidizing upland soil sluster a. Environ Microbiol. 2018;20:1016-29.

34. Rochman FF, Kwon M, Khadka R, Tamas I, Lopez-Jauregui AA, Sheremet A, et al. Novel copper-containing membrane monooxygenases (CuMMOs) encoded by alkane-utilizing Betaproteobacteria. ISME J. 2020;14:714-26.

35. Holmes AJ, Costello A, Lidstrom ME, Murrell JC. Evidence that participate methane monooxygenase and ammonia monooxygenase may be evolutionarily related. FEMS Microbiol Lett. 1995;132:203-8.

36. Khadka R, Clothier L, Wang L, Lim CK, Klotz MG, Dunfield PF. Evolutionary history of copper membrane monooxygenases. Front Microbiol. 2018;9:2493.

37. Ross MO, Rosenzweig AC. A tale of two methane monooxygenases. J Biol Inorg Chem. 2017;22:307-19.

38. Lancaster KM, Caranto JD, Majer SH, Smith MA. Alternative bioenergy: updates to and challenges in nitrification metalloenzymology. Joule. 2018;2:421-41.

39. Hooper AB, Vannelli T, Bergmann DJ, Arciero DM. Enzymology of the oxidation of ammonia to nitrite by bacteria. Antonie van Leeuwenhoek. 1997;71:59-67.

40. Zahn JA, DiSpirito AA. Membrane-associated methane monooxygenase from Methylococcus capsulatus (Bath). J Bacteriol. 1996;178:1018-29.

41. Zou B, Huang Y, Zhang PP, Ding XM, Op den Camp HJM, Quan ZX. Horizontal gene transfer of genes encoding copper-containing membrane-bound monooxygenase (CUMMO) and soluble di-iron monooxygenase (SDIMO) in ethane- and propaneoxidizing Rhodococcus bacteria. Appl Environ Microbiol. 2021;87:e00227-21.

42. Coleman NV, Le NB, Ly MA, Ogawa HE, McCarl V, Wilson NL, et al. Hydrocarbon monooxygenase in Mycobacterium: recombinant expression of a member of the ammonia monooxygenase superfamily. ISME J. 2012;6:171-82.

43. Lund J, Woodland MP, Dalton H. Electron transfer reactions in the soluble methane monooxygenase of Methylococcus capsulatus (Bath). Eur J Biochem. 1985; 147:297-305.

44. Hanson RS, Hanson TE. Methanotrophic bacteria. Microbiological Rev. 1996;60:439-71.

45. Dedysh SN, Knief C, Dunfield PF. Methylocella species are facultatively methanotrophic. J Bacteriol. 2005;187:4665-70.

46. Fosdike WLJ, Smith TJ, Dalton H. Adventitious reactions of alkene monooxygenase reveal common reaction pathways and component interactions among bacterial hydrocarbon oxygenases. FEBS J. 2005;272:2661-9.

47. Suzuki I, Kwok S-C, Dular U. Competitive inhibition of ammonia oxidation in Nitrosomonas europaea by methane, carbon monoxide or methanol. FEBS Lett. 1976;72:117-20. 
48. Keener WK, Arp DJ. Kinetic studies of ammonia monooxygenase inhibition in Nitrosomonas europaea by hydrocarbons and halogenated hydrocarbons in an optimized whole-cell assay. Appl Environ Microbiol. 1993;59:2501-10.

49. Hyman MR, Murton IB, Arp DJ. Interaction of ammonia monooxygenase from Nitrosomonas europaea with alkanes, alkenes, and alkynes. Appl Environ Microbiol. 1988:54:3187-90.

50. Hubley JH, Thomson AW, Wilkinson JF. Specific inhibitors of methane oxidation in Methylosinus trichosporium. Arch Microbiol. 1975;102:199-202.

51. Prior SD, Dalton H. The effect of copper ions on membrane content and methane monooxygenase activity in methanol-grown cells of Methylococcus capsulatus (Bath). Microbiology. 1985;131:155-63.

52. Pham MD, Lin YP, Van Vuong $Q$, Nagababu $P$, Chang BT, Ng KY, et al. Inactivation of the particulate methane monooxygenase (pMMO) in Methylococcus capsulatus (Bath) by acetylene. Biochimi Biophys Acta. 2015;1854:1842-52.

53. Arp DJ, Stein LY. Metabolism of inorganic N compounds by ammonia-oxidizing bacteria. Crit Rev Biochem Mol Biol. 2003;38:471-95.

54. Taylor AE, Taylor K, Tennigkeit B, Palatinszky M, Stieglmeier M, Myrold DD, et al. Inhibitory effects of $C_{2}$ to $C_{10}$ 1-alkynes on ammonia oxidation in two Nitrososphaera species. Appl Environ Microbiol. 2015;81:1942-8.

55. Prior SD, Dalton $\mathrm{H}$. Acetylene as a suicide substrate and active site probe for methane monooxygenase from Methylococcus capsulatus (Bath). FEMS Microbiol Lett. 1985;29:105-9.

56. Lontoh S, DiSpirito AA, Krema CL, Whittaker MR, Hooper AB, Semrau JD. Differential inhibition in vivo of ammonia monooxygenase, soluble methane monooxygenase and membrane-associated methane monoxygenase by phenylacetylene. Environ Microbiol. 2000;2:485-94.

57. Cole JR, Wang Q, Cardenas E, Fish J, Chai B, Farris RJ, et al. The Ribosomal Database Project: improved alignments and new tools for rRNA analysis. Nucleic Acids Res. 2009;37:D141-5.

58. Koops H-P, Böttcher B, Möller UC, Pommerening-Röser A, Stehr G. Classification of eight new species of ammonia-oxidizing bacteria: Nitrosomonas communis $\mathrm{sp}$. nov., Nitrosomonas ureae sp. nov., Nitrosomonas aestuarii sp. nov., Nitrosomonas marina sp. nov., Nitrosomonas nitrosa sp. nov., Nitrosomonas eutropha sp. nov., Nitrosomonas oligotropha sp. nov. and Nitrosomonas halophila sp. nov. J Gen Microbiol. 1991;137:1689-99.

59. Norton JM, Klotz MG, Stein LY, Arp DJ, Bottomley PJ, Chain PSG, et al. Complete genome sequence of Nitrosospira multiformis, an ammonia-oxidizing bacterium from the soil environment. Appl Environ Microbiol. 2008;74:3559-72.

60. Kits KD, Sedlacek C, Lebedeva EV, Han P, Bulaev A, Pjevac $P$, et al. Kinetic analysis of a complete nitrifier reveals an oligotrophic lifestyle. Nature. 2017;549:269-72.

61. Mundinger AB, Lawson $C E$, Jetten MSM, Koch $H$, Lücker S. Cultivation and transcriptional analysis of a canonical Nitrospira under stable growth conditions. Front Microbiol. 2019;10:1325.

62. Kip N, Ouyang W, van Winden J, Raghoebarsing A, van Niftrik L, Pol A, et al. Detection, isolation, and characterization of acidophilic methanotrophs from Sphagnum mosses. Appl Environ Microbiol. 2011;77:5643-54.

63. Dedysh SN, Berestovskaya YY, Vasylieva LV, Belova SE, Khmelenina VN, Suzina $\mathrm{NE}$, et al. Methylocella tundrae sp. nov., a novel methanotrophic bacterium from acidic tundra peatlands. Int J Syst Evolut Microbiol. 2004;54:151-6.

64. Ghashghavi M, Belova SE, Bodelier PLE, Dedysh SN, Kox MAR, Speth DR, et al. Methylotetracoccus oryzae Strain $\mathrm{C} 50 \mathrm{C} 1$ is a novel type lb gammaproteobacterial methanotroph adapted to freshwater environments. mSphere. 2019;4:e00631-18.

65. Dubbels BL, Sayavedra-Soto LA, Bottomley PJ, Arp DJ. Thauera butanivorans sp. nov., a $C_{2}-C_{9}$ alkane-oxidizing bacterium previously referred to as "Pseudomonas butanovora". Int J Syst Evolut Microbiol. 2009;59:1576-8.

66. Hogendoorn C, Pol A, Picone N, Cremers G, van Alen TA, Gagliano AL, et al. Hydrogen and carbon monoxide-utilizing Kyrpidia spormannii species from Pantelleria island, Italy. Front Microbiol. 2020;11:951.

67. Sakoula D, Koch H, Frank J, Jetten MSM, van Kessel MAHJ, Lücker S. Enrichment and physiological characterization of a novel comammox Nitrospira indicates ammonium inhibition of complete nitrification. ISME J. 2021;15:1010-24.

68. Taylor S, Ninjoor V, Dowd DM, Tappel AL. Cathepsin B2 measurement by sensitive fluorometric ammonia analysis. Anal Biochem. 1974;60:153-62.

69. Griess P. Bemerkungen zu der Abhandlung der HH. Weselsky und Benedikt "Ueber einige Azoverbindungen". Ber der Dtsch Chemischen Ges. 1879;12:426-8.

70. Miranda KM, Espey MG, Wink DA. A rapid, simple spectrophotometric method for simultaneous detection of nitrate and nitrite. Nitric Oxide. 2001;5:62-71.

71. Daims $\mathrm{H}$. Use of fluorescence in situ hybridization and the daime image analysis program for the cultivation-independent quantification of microorganisms in environmental and medical samples. Cold Spring Harb Protoc. 2009;2009:pdb. prot5253.

72. Stoecker K, Dorninger C, Daims H, Wagner M. Double labeling of oligonucleotide probes for fluorescence in situ hybridization (DOPE-FISH) improves signal intensity and increases rRNA accessibility. Appl Environ Microbiol. 2010;76:922-6.
73. Slot JW, Geuze HJ. Cryosectioning and immunolabeling. Nat Protoc. 2007;2:2480-91.

74. Tokuyasu KT. A technique for ultracryotomy of cell suspensions and tissues. J Cell Biol. 1973;57:551-65.

75. Liou W, Geuze HJ, Slot JW. Improving structural integrity of cryosections for immunogold labeling. Histochemistry Cell Biol. 1996;106:41-58.

76. Daims $H$, Lücker $S$, Wagner $M$. daime, a novel image analysis program for microbial ecology and biofilm research. Environ Microbiol. 2006;8:200-13.

77. Daims H, Wagner M. Quantification of uncultured microorganisms by fluorescence microscopy and digital image analysis. Appl Microbiol Biotechnol. 2007;75:237-48.

78. Bushnell B. BBMap. 2014, https://jgi.doe.gov/data-and-tools/bbtools/. Accessed on 12-12-2017.

79. Nurk S, Meleshko D, Korobeynikov A, Pevzner PA. metaSPAdes: a new versatile metagenomic assembler. Genome Res. 2017;27:824-34.

80. Li H, Durbin R. Fast and accurate long-read alignment with Burrows-Wheeler transform. Bioinformatics. 2010;26:589-95.

81. Li H, Handsaker B, Wysoker A, Fennell T, Ruan J, Homer N, et al. The sequence alignment/map format and SAMtools. Bioinformatics. 2009;25:2078-9.

82. Graham ED, Heidelberg JF, Tully BJ. BinSanity: unsupervised clustering of environmental microbial assemblies using coverage and affinity propagation. PeerJ. 2017;5:e3035.

83. Lu YY, Chen T, Fuhrman JA, Sun F. COCACOLA: binning metagenomic contigs using sequence COmposition, read CoverAge, CO-alignment and paired-end read LinkAge. Bioinformatics. 2017;33:791-8.

84. Alneberg J, Bjarnason BS, de Bruijn I, Schirmer M, Quick J, ljaz UZ, et al. Binning metagenomic contigs by coverage and composition. Nat Methods. 2014;11:1144-6.

85. Wu YW, Simmons BA, Singer SW. MaxBin 2.0: an automated binning algorithm to recover genomes from multiple metagenomic datasets. Bioinformatics. 2016:32:605-7.

86. Kang DD, Froula J, Egan R, Wang Z. MetaBAT, an efficient tool for accurately reconstructing single genomes from complex microbial communities. PeerJ. 2015;3:e1165.

87. Sieber CMK, Probst AJ, Sharrar A, Thomas BC, Hess M, Tringe SG, et al. Recovery of genomes from metagenomes via a dereplication, aggregation and scoring strategy. Nat Microbiol. 2018;3:836-43.

88. Parks DH, Imelfort M, Skennerton CT, Hugenholtz P, Tyson GW. CheckM: assessing the quality of microbial genomes recovered from isolates, single cells, and metagenomes. Genome Res. 2015;25:1043-55.

89. Parks DH, Chuvochina M, Waite DW, Rinke C, Skarshewski A, Chaumeil P-A, et al. A standardized bacterial taxonomy based on genome phylogeny substantially revises the tree of life. Nat Biotechnol. 2018;36:996.

90. Eren AM, Esen ÖC, Quince C, Vineis JH, Morrison HG, Sogin ML, et al. Anvi'o: an advanced analysis and visualization platform for 'omics data. PeerJ. 2015;3: e1319.

91. Wick RR, Schultz MB, Zobel J, Holt KE. Bandage: interactive visualization of de novo genome assemblies. Bioinformatics. 2015;31:3350-2.

92. Altschul SF, Gish W, Miller W, Myers EW, Lipman DJ. Basic local alignment search tool. J Mol Biol. 1990;215:403-10.

93. Shaffer M, Borton MA, McGivern BB, Zayed AA, La Rosa Sabina L, Solden LM, et al. DRAM for distilling microbial metabolism to automate the curation of microbiome function. Nucleic Acids Res. 2020;48:8883-900.

94. Olm MR, Brown CT, Brooks B, Banfield JF. dRep: a tool for fast and accurate genomic comparisons that enables improved genome recovery from metagenomes through de-replication. ISME J. 2017;11:2864-8.

95. Hyatt D, Chen G-L, LoCascio PF, Land ML, Larimer FW, Hauser LJ. Prodigal: prokaryotic gene recognition and translation initiation site identification. BMC Bioinforma. 2010;11:119.

96. Eddy SR. Accelerated profile HMM searches. PLOS Computational Biol. 2011;7: e1002195.

97. El-Gebali S, Mistry J, Bateman A, Eddy SR, Luciani A, Potter SC, et al. The Pfam protein families database in 2019. Nucleic Acids Res. 2018;47:D427-32.

98. Katoh K, Standley DM. MAFFT multiple sequence alignment software version 7: improvements in performance and usability. Mol Biol Evolution. 2013;30:772-80.

99. Stamatakis A. RAxML version 8: a tool for phylogenetic analysis and postanalysis of large phylogenies. Bioinformatics. 2014;30:1312-3.

100. R Core Team. R: a language and environment for statistical computing, version 3.5.3. Vienna, Austria: R Foundation for Statistical Computing; 2019.

101. Wickham H, Averick M, Bryan J, Chang W, McGowan LDA, François R, et al. Welcome to the Tidyverse. J Open Source Softw. 2019;4:1686.

102. Wright CL, Schatteman A, Crombie AT, Murrell JC, Lehtovirta-Morley LE. Inhibition of ammonia monooxygenase from ammonia-oxidizing Archaea by linear and aromatic alkynes. Appl Environ Microbiol. 2020;86:e02388-19. 
103. Hamamura N, Storfa RT, Semprini L, Arp DJ. Diversity in butane monooxygenases among butane-grown bacteria. Appl Environ Microbiol. 1999;65:4586-93.

104. Geets J, Boon N, Verstraete W. Strategies of aerobic ammonia-oxidizing bacteria for coping with nutrient and oxygen fluctuations. FEMS Microbiol Ecol. 2006;58:1-13.

105. Kitmitto A, Myronova N, Basu P, Dalton H. Characterization and structural analysis of an active particulate methane monooxygenase trimer from Methylococcus capsulatus (Bath). Biochemistry. 2005;44:10954-65.

106. Fiencke C, Bock E. Immunocytochemical localization of membrane-bound ammonia monooxygenase in cells of ammonia oxidizing bacteria. Arch Microbiol. 2006;185:99-106.

107. Murray RGE, Watson SW. Structure of Nitrosocystis oceanus and comparison with Nitrosomonas and Nitrobacter. J Bacteriol. 1965;89:1594-609.

108. Smith U, Ribbons DW, Smith DS. The fine structure of Methylococcus capsulatus. Tissue Cell. 1970;2:513-20.

109. Zhou Z, Liu Y, Pan J, Cron BR, Toner BM, Anantharaman K, et al. Gammaproteobacteria mediating utilization of methyl-, sulfur- and petroleum organic compounds in deep ocean hydrothermal plumes. ISME J. 2020;14:3136-48.

110. Karthikeyan S, Rodriguez-R LM, Heritier-Robbins P, Kim M, Overholt WA, Gaby $\mathrm{JC}$, et al. "Candidatus Macondimonas diazotrophica", a novel gammaproteobacterial genus dominating crude-oil-contaminated coastal sediments. ISME J. 2019;13:2129-34.

111. Palomo A, Pedersen AG, Fowler SJ, Dechesne A, Sicheritz-Pontén T, Smets BF. Comparative genomics sheds light on niche differentiation and the evolutionary history of comammox Nitrospira. ISME J. 2018;12:1779-93.

112. Daims $H$, Lücker $S$, Wagner $M$. A new perspective on microbes formerly known as nitrite-oxidizing bacteria. Trends Microbiol. 2016;24:699-712.

113. Shah VK, Davis LC, Brill WJ. Nitrogenase: VI. Acetylene reduction assay: dependence of nitrogen fixation estimates on component ratio and acetylene concentration. Biochim Biophys Acta. 1975;384:353-9.

114. Whidbey C, Sadler NC, Nair RN, Volk RF, DeLeon AJ, Bramer LM, et al. A probeenabled approach for the selective isolation and characterization of functionally active subpopulations in the gut microbiome. J Am Chem Soc. 2019;141:42-7.

115. Whidbey C, Wright AT. Activity-based protein profiling-enabling mltimoda fnctional studies of microbial communities. In: Cravatt BF, Hsu K-L, Weerapana E, editors. Activity-based protein profiling. Cham: Springer International Publishing; 2019. p. 1-21.

116. Stein LY, Arp DJ, Hyman MR. Regulation of the synthesis and activity of ammonia monooxygenase in Nitrosomonas europaea by altering $\mathrm{pH}$ to affect $\mathrm{NH}_{3}$ availability. Appl Environ Microbiol. 1997;63:4588-92.

117. Pjevac $P$, Schauberger $C$, Poghosyan L, Herbold CW, van Kessel MAHJ, Daebeler A. et al. AmoA-targeted polymerase chain reaction primers for the specific detection and quantification of comammox Nitrospira in the environment. Front Microbiol. 2017;8:1508

118. Rotthauwe $\mathrm{JH}$, Witzel KP, Liesack $\mathrm{W}$. The ammonia monooxygenase structural gene $a m o A$ as a functional marker: molecular fine-scale analysis of natural ammonia-oxidizing populations. Appl Environ Microbiol. 1997;63:4704-12.

119. Ghashghavi M, Jetten MSM, Lüke C. Survey of methanotrophic diversity in various ecosystems by degenerate methane monooxygenase gene primers. AMB Express. 2017;7:162.

120. Pinto AJ, Marcus DN, ljaz UZ, Bautista-de Lose Santos QM, Dick GJ, Raskin L. Metagenomic evidence for the presence of comammox Nitrospira-like bacteria in a drinking water system. mSphere. 2016;1:e00054-15.

121. Palomo A, Jane Fowler S, Gulay A, Rasmussen S, Sicheritz-Ponten T, Smets BF. Metagenomic analysis of rapid gravity sand filter microbial communities suggests novel physiology of Nitrospira spp. ISME J. 2016;10:2569-81.

122. Bartelme R, McLellan S, Newton R. Freshwater recirculating aquaculture system operations drive biofilter bacterial community shifts around a stable nitrifying consortium of ammonia-oxidizing archaea and comammox Nitrospira. Front Microbiol. 2017;8:101.

123. Wang J-G, Xia F, Zeleke J, Zou B, Rhee S-K, Quan Z-X. An improved protocol with a highly degenerate primer targeting copper-containing membrane-bound monooxygenase genes for community analysis of methane- and ammoniaoxidizing bacteria. FEMS Microbiol Ecol. 2017;93:fiw244.

124. Fowler SJ, Palomo A, Dechesne A, Mines PD, Smets BF. Comammox Nitrospira are abundant ammonia oxidizers in diverse groundwater-fed rapid sand filter communities. Environ Microbiol. 2018:20:1002-15.

125. Xia F, Wang JG, Zhu T, Zou B, Rhee SK, Quan ZX. Ubiquity and diversity of complete ammonia oxidizers (comammox). Appl Environ Microbiol. 2018;84:e01390-18.
126. Fujitani H, Kumagai A, Ushiki N, Momiuchi K, Tsuneda S. Selective isolation of ammonia-oxidizing bacteria from autotrophic nitrifying granules by applying cell-sorting and sub-culturing of microcolonies. Front Microbiol. 2015;6:1159.

127. Morgan-Sagastume F, Larsen P, Nielsen JL, Nielsen PH. Characterization of the loosely attached fraction of activated sludge bacteria. Water Res. 2008;42:843-54.

128. Mcllroy SJ, Albertsen M, Andresen EK, Saunders AM, Kristiansen R, StokholmBjerregaard $\mathrm{M}$, et al. "Candidatus Competibacter"-lineage genomes retrieved from metagenomes reveal functional metabolic diversity. ISME J. 2013;8:613-24.

129. Mcllroy SJ, Nittami T, Kanai E, Fukuda J, Saunders AM, Nielsen PH. Re-appraisal of the phylogeny and fluorescence in situ hybridization probes for the analysis of the Competibacteraceae in wastewater treatment systems. Environ Microbiol Rep. 2015;7:166-74.

\section{ACKNOWLEDGEMENTS}

The authors would like to thank C. Hogendoorn, M. Ghashghavi, M.A.R. Kox, C.J. Sedlacek, and L. Lehtovirta-Morley for the generous provision of biomass, and T. van Alen and $\mathrm{H}$. Harhangi for technical assistance. We are grateful to $\mathrm{H}$. Koch, M. Wagner, and $\mathrm{H}$. Daims for helpful discussions. DS and MSMJ were supported by the European Research Council (ERC Advanced Grant Ecomom 339880) and JF, GJS, LFMK, PB, MAHJvK, and SL by the Netherlands Organisation for Scientific Research (NWO Gravitation Grant SIAM 024.002.002, 016.Veni.192.062 and 016.Vidi.189.050).

\section{AUTHOR CONTRIBUTIONS}

SL conceived the presented research. DS, MAHJvK, MSMJ, and SL planned research. SL and MAHJvK supervised the project. DS, GS, RJM, JF, LK, and PB executed experiments and analyzed data. DS, GS, MAHJvK, and SL wrote the paper. All authors discussed results and commented on the manuscript.

\section{COMPETING INTERESTS}

The authors declare no competing interests.

\section{ADDITIONAL INFORMATION}

Supplementary information The online version contains supplementary material available at https://doi.org/10.1038/s41396-021-01144-0.

Correspondence and requests for materials should be addressed to Dimitra Sakoula or Sebastian Lücker.

Reprints and permission information is available at http://www.nature.com/ reprints

Publisher's note Springer Nature remains neutral with regard to jurisdictional claims in published maps and institutional affiliations.

(i) Open Access This article is licensed under a Creative Common adaptation, distribution and reproduction in any medium or format, as long as you give appropriate credit to the original author(s) and the source, provide a link to the Creative Commons license, and indicate if changes were made. The images or other third party material in this article are included in the article's Creative Commons license, unless indicated otherwise in a credit line to the material. If material is not included in the article's Creative Commons license and your intended use is not permitted by statutory regulation or exceeds the permitted use, you will need to obtain permission directly from the copyright holder. To view a copy of this license, visit http://creativecommons. org/licenses/by/4.0/.

(c) The Author(s) 2021 